\title{
Primate heart regeneration via migration and fibroblast repulsion by human heart progenitors
}

Christine Schneider $^{1 \pi}$, Kylie S. Foo ${ }^{2 \pi}$, Maria Teresa De Angelis ${ }^{3 \pi}$, Gianluca Santamaria ${ }^{3 \pi}$, Franziska Reiter $^{1}$, Tatjana Dorn ${ }^{3}$, Andrea Bähr ${ }^{1}$, Yat Long Tsoi ${ }^{2}$, Petra Hoppmann ${ }^{1}$, Ilaria My ${ }^{1}$, Anna Meier ${ }^{3}$, Victoria Jurisch ${ }^{1}$, Nadja Hornaschewitz ${ }^{1}$, Sascha Schwarz ${ }^{6}$, Kun Lu ${ }^{7}$, Roland Tomasi ${ }^{7}$, Stefanie Sudhop ${ }^{6}$, Elvira Parrotta ${ }^{8}$, Marco Gaspari ${ }^{8}$, Giovanni Cuda ${ }^{8}$, Nikolai Klymiuk ${ }^{1,9}$, Andreas Dendorfer ${ }^{7,9}$, Markus Krane ${ }^{5,9}$, Christian Kupatt ${ }^{1,9}$, Daniel Sinnecker ${ }^{1,9}$, Alessandra Moretti ${ }^{3,9 *}$, Kenneth R. Chien ${ }^{2 *}$, and Karl-Ludwig Laugwitz $z^{1,9 *}$

${ }^{1}$ Medical Department I - Cardiology, Angiology, Pneumology, Klinikum rechts der Isar - Technical University of Munich, Ismaninger Strasse 22, 81675 Munich, Germany

${ }^{2}$ Department of Cell and Molecular Biology, Karolinska Institutet, SE-171 77 Stockholm, Sweden

3 Institute of Regenerative Medicine in Cardiology - Technical University of Munich, Ismaninger Strasse 22, 81675 Munich, Germany

${ }^{4}$ Department of Medicine, Karolinska Institutet, SE-171 77 Stockholm, Sweden

${ }^{5}$ Department of Cardiovascular Surgery, INSURE (Institute for Translational Cardiac Surgery), German Heart Center Munich - Technical University of Munich, Lazarettstrasse 36, 80636 Munich, Germany

${ }^{6}$ Center for Applied Tissue Engineering and Regenerative Medicine (CANTER), Munich University of Applied Sciences, Lothstrasse 34, 80335 Munich, Germany

7 Walter-Brendel-Centre of Experimental Medicine, University Hospital, LMU Munich, Marchioninistrasse 27, 81377 Munich, Germany

${ }^{8}$ Department of Experimental and Clinical Medicine, Medical School, University of Magna Grecia, Catanzaro, Italy

${ }^{9}$ DZHK (German Centre of Cardiovascular Research) - partner site Munich Heart Alliance, Munich, Germany

Word count: Manuscript 3866 words; Abstract 232 words

१ These authors contributed equally to this work

* Correspondence should be addressed to A.Mo., K.-L.L. and K.R.C.:

44 amoretti@mytum.de, laugwitz@mytum.de, and kenneth.chien@ki.se 
SUMMARY

Human heart regeneration is one of the most critical unmet clinical needs at a global level ${ }^{1}$. Muscular regeneration is hampered both by the limited renewing capacity of adult cardiomyocytes $^{2-4}$ and the onset of cardiac fibrosis ${ }^{5,6}$, resulting in reduced compliance of the tissue. Primate have proven to be ideal models for pluripotent stem cell strategies for heart regeneration, but unravelling specific approaches to drive cell migration to the site of injury and

52 inhibition of subsequent fibrosis have been elusive. Herein, by combining human cardiac

53 progenitor lineage tracing and single-cell transcriptomics in injured non-human primate heart

54 bio-mimics, we uncover the coordinated muscular regeneration of the primate heart via directed 55 migration of human ventricular progenitors to sites of injury, subsequent fibroblast repulsion 56 targeting fibrosis, and ultimate functional replacement of damaged cardiac muscle by 57 differentiation and electromechanical integration. Single-cell RNAseq captured distinct modes 58 of action, uncovering chemoattraction mediated by CXCL12/CXCR4 signalling and fibroblast 59 repulsion regulated by SLIT2/ROBO1 guidance in organizing cytoskeletal dynamics. Moreover, 60 transplantation of human cardiac progenitors into hypo-immunogenic CAG-LEA29Y 61 transgenic porcine hearts following injury proved their chemotactic response and their ability to generate a remuscularized scar without the risk of arrhythmogenesis in vivo. Our study

63 demonstrates that inherent developmental programs within cardiac progenitors are sequentially 64 activated in the context of disease, allowing the cells to sense and counteract injury. As such, 65 they may represent an ideal bio-therapeutic for functional heart rejuvenation. 
67 Whereas mammals do have the capacity to undergo endogenous cardiac regeneration during

68 development and shortly after birth $^{7}$, the regenerative capacity of the human heart in adulthood is

69 markedly low $^{3}$. The inability to replace lost myocardium is accompanied by extensive tissue

70 remodelling and fibrosis, leaving patients with cardiac disease vulnerable to heart failure. Although

71 numerous drugs and mechanical devices can moderately improve cardiac function, such approaches

72 do not replace lost cardiomyocytes (CM) or abolish fibrotic scar formation. Over the past decade, bio-

73 therapies have emerged as innovative strategies for heart repair ${ }^{8-11}$. Induction of endogenous CM

74 proliferation ${ }^{12-15}$, in vivo direct reprogramming of non-CMs to a cardiac fate ${ }^{16}$, and exogenous

75 transplantation of human pluripotent stem cell (hPSC)-derived $\mathrm{CMs}^{17}$ or cardiac progenitors ${ }^{18}$ have

76 been recently explored as potential approaches to generate de novo myocardium.

77 Studies in lower vertebrates, where robust cardiac regeneration occurs throughout life, have 78 demonstrated that endogenous heart repair is a highly coordinated process involving inter-lineage 79 communication, cellular de- and re-differentiation, migration, and extracellular matrix (ECM)

80 remodelling without fibrotic scarring ${ }^{19-22}$. Similar programs are the foundation of organ 81 morphogenesis and are inherent of embryonic cardiac progenitors. During heart development, defined 82 embryonic precursors, including first heart field (FHF) and second heart field (SHF), give rise to 83 distinct cardiac compartments and cell types ${ }^{23,24}$. While FHF cells, marked by HCN4 and NKX2.5, are 84 fated to differentiate early into CMs of the primitive heart tube, ISL1 ${ }^{+}$SHF has broader lineage 85 potential and its differentiation is preceded by an extensive proliferation and directed migration into 86 the forming myocardium ${ }^{25-27}$. We have recently reported the generation of an enriched pool of hiPSC87 derived $\mathrm{ISLl}^{+}$ventricular progenitors (HVPs), which can expand and differentiate into functional 88 ventricular CMs in vitro and in vivo ${ }^{28}$. Here, we sought to determine whether HVPs could effectively 89 provide primate heart regeneration by orchestrating and activating sequential programs of cardiac 90 development, ultimately leading to de novo myocardium formation and fibrotic scar-less healing.

\section{HVPs functionally repopulate a chronic injury model of non-human primate heart slices}

93 During cardiogenesis, heart progenitors develop in a three-dimensional (3D) micro-environment 94 incorporating important cues from myocardial architecture and electromechanical forces. To dissect 95 molecular steps of HVP-mediated cardiac repair at a single cell level, we established an ex vivo 3D 96 chimeric model where HVPs were exposed to the complex structural and molecular environment of 97 adult heart tissue from non-human primate (NHP). In customized bio-mimetic chambers ${ }^{29}$, native 98 NHP left ventricle (LV) slices ( $\sim 300 \mu \mathrm{m}$ thickness) were cultured under physiological preload (1 mN) 
and continuous stimulation ( $1 \mathrm{~Hz}$ pacing), allowing proper structural and functional preservation for up to 14 days (D) (Fig. 1a, b and Extended Data Fig. 1a). Subsequently, progressive tissue deterioration occurred, as indicated by the gradual loss of contractile force that coincided with increasing apoptotic CM death over time (Fig. 1b, c and Extended Data Fig. 1a, b), offering an ideal setting for investigating cell-based mechanisms of cardiac repair. $N K X 2.5^{\mathrm{eGFP} / w t}$ human embryonic stem cells (hESC) were coaxed towards ISL1/NKX2.5 expressing heart progenitors using our previously described two-step protocol that enriches for $\mathrm{HVPs}^{28}$, with small number of multipotent ISL1 $^{+}$precursors $^{30}$ (Fig. 1a and Extended Data Fig. 1c). After magnetic-activated cell sorting (MACS)-based depletion of undifferentiated hESCs, cells were seeded onto the NHP LV slices by standardized bioprinting (Extended Data Fig. 1c, d). eGFP expression regulated by the NKX2.5 locus allowed live tracing of HVPs and their derivative CMs within the NHP myocardium (Extended Data Fig. 1e, top). Labelling with EdU and activated caspase-3 (ClCasp3) indicated that eGFP ${ }^{+}$cells were highly proliferative during the first 2 weeks, but stopped expanding by D21 when eGFP- NHP-CMs underwent massive apoptosis (Fig. 1c and Extended Data Fig. 1f). This corresponded to the extensive differentiation towards CMs and downregulation of ISL1 expression (Extended Data Fig. 1g). Remarkably, heart slices gradually regained contractile force in the third week of co-culture, reaching $2 \mathrm{mN}$ force generation that was further maintained up to D50 (Fig. 1b and Extended Data Fig. 1e). Immunohistochemistry for atrial and ventricular muscle markers (MLC2a / MLC2v) revealed that the majority of $\mathrm{eGFP}^{+}$cells acquired a ventricular muscle identity over time (Fig. 1d). By D21, 19\% expressed both markers, indicative of CM entering the ventricular lineage, and $\sim 69 \%$ were already exclusively MLC2v positive, representing maturing ventricular CMs. The latter reached $\sim 81 \%$ by D50 (Fig. 1d). Moreover, at this stage, most $\mathrm{eGFP}^{+} / \mathrm{MLC} \mathrm{v}^{+} \mathrm{CMs}$ presented a rod-shape appearance with well-aligned myofibrils, structural characteristics of mature muscle cells (Fig. 1d). A small proportion of human cells expressing the endothelial marker CD31 were also detected $(\sim 14 \%$ on D21 and $\sim 7 \%$ on D50) (Fig. 1e), likely arising from multipotent precursors within the HVP pool.

To establish a molecular roadmap for HVP specification and maturation during heart repair, we profiled cells on D0 (before seeding on NHP LV slices) and eGFP ${ }^{+}$cells isolated on D3 and D21 of ex-vivo co-culture by single cell RNAseq (scRNAseq). We then integrated the data with our previously published scRNAseq dataset from D-3 of in-vitro differentiation ${ }^{31}$. Transcriptomes of over 1,615 cells were recovered. Unsupervised clustering analysis identified 7 distinct sub-populations; stage dependent clustering was evident for all samples (Fig. 1f). On D-3, corresponding to the time point of cardiac lineage commitment ${ }^{32}$, cells expressed high levels of genes typical of early cardiac 
131 mesodermal cells, such as EOMES, MESP1 and LGR5 (Extended Data Fig. 2a, b and Supplementary

132 Table 1). On D0, cells distributed into 4 distinct clusters: transcriptomes of early (KRT18/ID1), intermediate (KRT8/PRDX1), and proliferating (TOP2A/CCNB1-2) heart progenitor states as well as

134 cardiac mesenchymal cells (PLCE1/PPA1) were captured (Fig. 1f and Extended Data Fig. 2a, b).

135 Transcripts related to extracellular matrix organization (DCN/TIMP1/LUM/FN1/COL3A1) and ventricular muscle structure/maturation (MYL3/TTN/TNNC1/ACTC1/PLN) defined late eGFP ${ }^{+}$cells and ventricular CMs on D3 and D21 within the myocardial tissue, respectively (Fig. 1f and Extended Data Fig. 2a, b). In order to achieve a temporal resolution of cardiac fate decisions, we aligned cells captured at the various time points in pseudotime, a computational measure of the progress a cell makes along a certain differentiation trajectory ${ }^{33}$. The resulting trajectory began with cardiac mesodermal cells on D-3 followed by mesenchymal cells and early progenitors from D0 and then bi-

142 furcated into two lineages where endothelial-committed late progenitors on D3 and HVPs and their derivative CMs on D21 were allocated (Fig. 1g and Extended Data Fig. 2c).

Gene ontology (GO) enrichment analysis of differentially expressed genes (DEGs) in cells

145 from D0 to D21 revealed progressive activation of GO terms related to cardiac ventricular 146 morphogenesis and maturation, while signalling pathways relevant to the cardiac progenitor state, 147 such as extracellular matrix organization, cell cycle, and canonical Wnt signalling, were gradually 148 suppressed (Fig. 1h). Interestingly, genes upregulated in progenitors at the early time of co-culture 149 also associated with cell migration, cell projection organization, cytokine production, and response to 150 TGF $\beta$ (Fig. 1h), suggesting a specific sensing-reacting response of HVPs to the tissue environment. 151 Of note, vasculature development was likewise enriched, confirming the additional potential of some 152 early precursors to differentiate into vessels. To better define the level of maturation achieved by the 153 HVP-derived CMs, we integrated our data with a published scRNAseq dataset of in vivo human adult 154 ventricular muscle ${ }^{34}$ in pseudotime (Fig. 1i and Extended Data Fig. 2d). eGFP ${ }^{+}$cells on D21 partially 155 allocated together with adult ventricular CMs at the end of the differentiation trajectory and expressed 156 high levels of structural, functional, and metabolic genes characteristic of the adult state (Fig. 1i and 157 Extended Data Fig. 2d). Taken together, our single cell transcriptomic analyses allowed the 158 construction of a differentiation route through which early mesodermal cardiac progenitors generate 159 mature CMs in response to the signalling cues of a gradually dying myocardium. 

injury model

164 Embarking on the heart tissue slices ex vivo, we designed a model of acute cardiac injury to provoke tissue death and elucidate HVP properties in response to injury signals (Fig. 2 and Extended Data Fig. 3). Radiofrequency ablation (RFA) is routinely employed in patients with atrial fibrillation to terminate arrhythmogenic foci. We established $20 \mathrm{~W}$ for $15 \mathrm{sec}$ as efficient RFA conditions to destroy, in a standardized manner, a defined area of the cellular compartment within the NHP heart slices, leaving the extracellular matrix (ECM) as scaffold intact (Extended Data Fig. 3a). A progressive invasion of activated cardiac fibroblasts (CFs) expressing the discoidin domain receptor 2 (DDR2) as well as an increase of collagen type I deposition in the RFA-injured area were visible over time, with a complete scarring of the tissue by D21 (Extended Data Fig. 3b). In the first series of experiments, we seeded equal amounts of $N K X 2.5^{\mathrm{eGFP} / w t}$ HVPs or CMs onto bio-printed pluronic frames on one side of the NHP heart slices, generated RFA injury on the opposite side, and evaluated the cellular

175 response to the damage by live cell imaging of the eGFP signal (Fig. 2a). In contrast to CMs, HVPs 176 departed from their local seeding site and migrated in a directed manner towards the injured region, colonising it within 4 days (Fig. 2a and Extended Data Fig. 3c, d). By D15, HVPs had differentiated

178 into CMs and the RFA area appeared largely remuscularized, with new eGFP ${ }^{+} \mathrm{CMs}_{\text {being elongated }}$ 179 and showing aligned myofibrils with organized sarcomeres on D21 (Fig. 2b). A significant reduction 180 of scar volume was measured exclusively in HVP-treated heart slices and, consistently, contractile 181 function of the tissue was considerably improved (Fig. 2c, $d$ and Extended Data Fig. 3e). To assess the 182 potential of HVP-derived CMs to functionally integrate into the electromechanical syncytium after 183 injury, we performed real-time intracellular $\mathrm{Ca}^{2+}$ analysis comparing regions of interest (ROI) within 184 the damaged and native myocardium. In contrast to CM-treated heart slices, Fluo-4 fluorescence 185 clearly propagated through the injury area when HVPs had been applied, with differentiated HVPs 186 displaying $\left[\mathrm{Ca}^{2+}\right] \mathrm{i}$ oscillations similar to and synchronized with those in adjacent native NHP CMs 187 (Fig. 2e), indicating electromechanical integration of the HVP-derived CMs.

188 To further dissect the cellular and molecular mechanisms underlying the observed HVP directed migration towards the RFA injured tissue and the subsequent positive remodelling during the

190 scarring process, we first evaluated the dynamic cellular composition of the tissue around and at the 191 injury site over time. Immunofluorescence analysis indicated that, one day after RFA, activated 192 DDR2 $^{+}$NHP CFs were heavily populating the border zone and already reached the damaged area 193 before the human eGFP ${ }^{+}$HVPs; both cells coexisted in the injured and surrounding regions after 1 
194 week (Fig. 2f). Shortly after, the RFA site was predominantly colonized by human eGFP ${ }^{+}$cells and

195 the border zone from NHP DDR2 ${ }^{+}$CFs (Fig. 2f). These observations suggested that cell-cell 196 communication through chemokines or physical interaction between the host CFs and the human 197 progenitors might instruct HVP migration, differentiation, and scar remodelling.

\section{Chemotaxis of HVPs to sites of cardiac injury is mediated by CXCL12/CXCR4 signalling}

200 During development ISL1 ${ }^{+}$HVPs are highly migratory for heart tube extension and outflow tract 201 formation $^{23}$. To gain insights into the mode of HVP migration in our cardiac injury model, we first 202 performed a trans-well migration assay, where HVPs were placed on top of a permeable membrane and RFA-injured or uninjured NHP heart slices at the bottom (Extended Data Fig. 3f). A significantly boosted migration was observed in presence of RFA. Interestingly, while multiple, homogeneously distributed RFAs prompted HVPs to evenly migrate through the membrane, a directional migration towards the injured area was observed with a single isolated RFA (Extended Data Fig. 3f), indicating the production of a chemoattractant gradient specifically arising from NHP cells at the damaged area.

To further dissect the molecular programs for directed HVP chemotaxis and response, we profiled migrating eGFP ${ }^{+}$cells (at 24 hours; 485 cells) and arriving $\mathrm{eGFP}^{+}$cells at the RFA injury (at 48 hours; 269 cells) as well as eGFP' tissue resident host cells (315 cells) by scRNAseq (Extended Data Fig. 4a). Cells were embedded in low-dimensional space using UMAP followed by unsupervised 213 (Extended Data Fig. 4a, b and Supplementary Table 1). Cluster 1 and 4 belonged to the NHP cell 214 group and mapped to CFs and monocyte/macrophages, respectively. Human cells formed the other 2 215 major cell groups. One contained 4 clusters, which were classified as early HVPs (expressing high 216 levels of metabolic genes as MBOAT1, UQCRQ, and MT-ND1,2,4,5,6, but lacking expression of CM 217 transcripts; cluster 0), activated HVPs (LAMA5, FLRT2, and TNC; cluster 2), proliferating HVPs 218 (TOP2A, CDC20, and CCNB2; cluster 5), and early ventricular CMs (MYH6, MYL3, TNNC1; cluster 219 6). The second comprised of a homogeneous population of HVPs (cluster 3) characterized by high 220 expression of genes involved in chemotaxis (NRP1, CCL2-19-21, CXCL2-6-8-12, ITGB1, WASF1, 221 RPS4X, INPPL1), an unique gene signature not captured before. GO analysis of DEGs between 222 cluster 3 and the other HVP clusters identified enrichment of terms related to cell motility, 223 chemotaxis, actin filament organization, axon guidance cues, and ECM organisation, (Extended Data 224 Fig. 4c), supporting the migratory feature of this cell population. We further directly characterized the intercellular communication signals between HVPs and NHP cardiac cells by performing an in silico 
226 single cell receptor-ligand pairing screen. We found over-representation of a significant pairing of 227 CXCL12 as ligand with several membrane receptors, including CXCR4, SDC4, ITGB1, and ACKR3 228 (Fig. 2g). While CXCL12, SDC4, and ITGB1 were expressed in both HVPs and NHP fibroblasts, the 229 CXCR4 and ACKR3 receptors were highly enriched in the HVPs (Extended Data Fig. 4d). Trans-well 230 migration assays under gain- and loss-of-function conditions demonstrated that HVPs exhibited 231 enhanced migratory behaviour under CXCL12 as chemoattractant, which was specifically reduced in 232 presence of blocking antibodies for CXCR4 and SDC4 (Fig. 2h). Notably, binding of CXCL12 to 233 SDC4 is known to facilitate its presentation to the CXCR4 receptor ${ }^{35}$. Collectively, our data support 234 the model that HVPs expressing CXCR4 sense CXCL12 secreted by CFs at the injury site as a 235 chemoattracting signal to repopulate the damaged myocardial compartment. A similar, chemokine236 controlled deployment of SHF cells has been identified as intra-organ crosstalk between progenitors 237 and FHF CMs during mouse cardiogenesis ${ }^{36}$, suggesting that migration programs that are functional 238 during cardiac development are re-activated in HVPs during organ regeneration.

Distinct dynamical cellular states underlie HVP regenerative potential upon tissue injury

241 To capture all transition cell types and analyse the stepwise process of HVP-mediated cardiac repair, 242 we integrated all scRNAseq data of HVPs on D0, 24h and 48h after RFA injury, as well as HVP243 derived CMs on D21 co-culture (2,114 cells) and generated a diffusion map of tissue damage-induced 244 cardiac differentiation (Fig. 3a, b). Heat-mapping of gene expression with cells ordered in the 245 trajectory revealed a temporal sequence of gene expression events and identified cells at intermediate 246 stages of injury sensing and injury response (Extended Data Fig. 5a). Dot plotting illustrated gene 247 signature shifts among the different stages (Fig. 3c). In the first $24 \mathrm{~h}$ after injury, HVPs "sense" the 248 tissue damage and activate gene programs for ECM remodelling (e.g. COL6A1, ADAMTS9, FLRT2), 249 secretion and response to cytokine (SPP1, STX8, TGFBI, IL6ST), as well as initiation of migration 250 (PLAT). Subsequently (48h), they upregulate genes typical of migratory cells, including 251 chemoattraction signalling genes (PLXNA2, CMTM3, and CXCL12), cell motility genes (SNAI1, 252 SNAI2, FAT1, and TIMP1), and transcripts of cytoskeleton organization (ARPC2) as well as axon 253 guidance (SLIT2, NFIB, and UNC5B) and cell projection (RGS2, THY1, and ITGA1). In this migratory 254 state, gene signatures of secretion (COPB2, VPS35, and SPTBN1) and cardiac muscle differentiation 255 (VCAM1, MHY6, PALLD, and TMOD1) become increasingly important as counteracting response to 256 injury (Fig. 3c). Indeed, mass spectrometry analysis of supernatants from NHP heart slices $48 \mathrm{~h}$ after 257 RFA injury revealed a significant upregulation of secreted proteins in the presence of HVPs 
258 (Extended Data Fig. 5b). Interestingly, the majority of them are involved in ECM organization (e.g. 259 HSPG2, SPARC, FN1) and fibrotic/inflammation response (e.g. FSTL1, PRDX1, SPTAN1), 260 reinforcing the capability of HVPs to influence scar remodelling.

\section{SLIT2/ROBO1 mediates activated fibroblast repulsion and reduces scar formation}

263 CFs play an essential role in heart development and repair. During heart regeneration in zebrafish, 264 activation of resident fibroblasts support CM growth and maturation through secretion of specific 265 ECM components ${ }^{2,37}$. To further investigate the temporal and spatial direct crosstalk between CFs and 266 HVPs in our ex vivo cardiac injury model, we isolated CFs from native NHP hearts, stably expressed 267 dsRed by lentiviral transduction, and performed live imaging during monolayer co-culture with NKX2.5 $5^{\mathrm{eGFP} / w t}$ HVPs. RFA injury (20W for $7 \mathrm{sec}$ ) was performed on one site of the $\mathrm{dsRed}^{+} \mathrm{CF}$ monolayer and seeding of $N K X 2.5^{\mathrm{eGFP} / w t}$ HVPs on the other (Fig. 3d). Similar to the native tissue, the first cells invading the injured area were dsRed ${ }^{+} \mathrm{CFs}$, followed by eGFP ${ }^{+}$HVPs within 5 days (Fig. 3d). Remarkably, while HVPs were directly chemoattracted to the injury, CFs appeared dynamically repelled at the contact sites with migrating HVPs (Fig. 3e and Extended Data Movie 1). Live cell tracking of more than 100 cells over 3 days demonstrated that the majority of CFs, after interacting with HVPs, indeed moved actively away from the HVP migratory path and were repelled from the injured area when the HVPs started to densely populate it on D8 (Fig. 3e). Immunocytochemistry of filamentous $(\mathrm{F})$-actin revealed a specific retraction of cell protrusions precisely occurring at cellular contact sites with the HVPs (Extended Data Fig. 6a), suggesting that the latter possibly control actin dynamics of CFs at the interaction sites. Given the detected upregulation of genes involved in axon guidance in the migratory HVP state, including SLIT2, we postulated that SLIT2/ROBO1 signalling, a known repulsive guidance cues for neuronal axons ${ }^{38}$, might control HVP-mediated CF repulsion by regulating cytoskeletal organization and cell motion. Co-immunofluorescence analysis demonstrated expression of both SLIT2 ligand and ROBO1 receptor in migrating HVPs on D3, while no signal was detected in the surrounding CFs (Fig. 3f). On D8, however, co-localization of SLIT2 and ROBO1 was observed mainly at the membrane of repulsed CFs, with enriched SLIT2 signal at the contact sites with the HVPs (Fig. 3f). Quantitative RT-PCR confirmed that SLIT2 was produced by the HVPs and ROBO1 was expressed in both cell types at the stage of CF repulsion (Extended Data Fig. 6b). Lossof-function experiments using an antibody blocking ROBO1 signalling substantiated that, under ROBO1 inhibition, HVPs failed to induce actin polymerization and lamellipodia formation in the interacting CFs, leading to reduced CF motility and lack of repulsion (Fig. 3g, h and Extended Data 
Fig. 6c). No effects were observed in the distant CFs (Extended Dada Fig. 6c). Conversely, treatment with recombinant human SLIT2 enhanced F-actin content and membrane protrusions specifically in CFs communicating with HVPs (Extended Data Fig. 6d), resulting in enhanced repulsion (Fig. 3h).

\section{HVPs regenerate injured porcine myocardium in vivo without inducing arrhythmias}

295 To investigate the full regenerative potential of HVPs, we performed allogeneic in vivo transplantation 296 experiments in genetic modified pigs ubiquitously expressing LEA29Y, a human CTLA4-Ig 297 derivative that blunts systemic $\mathrm{T}$ cell response ${ }^{39}$. This immuno-compromised model offers an ideal 298 setting for testing human cell therapies in xenotransplantation approaches, enabling improved graft survival. Two epicardial RFA injuries $(25 \mathrm{~W}$ for $7 \mathrm{sec})$ were induced afar in the anterior heart wall and $6 \times 10^{7} N K X 2.5^{\mathrm{eGFP} / w t} \mathrm{HVPs}$ were injected $\sim 1 \mathrm{~cm}$ apart from one damaged site, while the other served as control (Fig. 4a and Extended Data Movie 2). Morphological assessment of RFA-induced tissue damage in freshly isolated wild-type porcine hearts demonstrated consistent size of myocardial injury (Fig. 4b, c). LEA29Y animals were treated daily with methylprednisolone and tacrolimus and euthanized under full anaesthesia on D3 $(n=1)$, D5 $(n=2)$, and D14 $(n=2)$ post-transplantation. None showed any macroscopic signs of tumour formation (Extended Data Fig. 7a). Immunohistology on D3 documented a directed, guided migration of eGFP ${ }^{+}$HVPs towards the RFA-injured area (Fig. 4d). On $\mathrm{D} 5$, eGFP ${ }^{+}$cells reached the RFA site, appeared mainly in clusters, and repopulated $6.3 \pm 0.6 \%$ of the scar (Fig. 4d, f). No significant difference in scar volume was measured at this time point between HVP-populated and control scars (Fig. 4e). However, 2 weeks after injury, eGFP ${ }^{+}$cells constituted $21.0 \pm 2.9 \%$ of the injured area and scar volume was significantly reduced to half compared to control

312 the depth of the analysed plane, denoting the highest concentration of $\mathrm{eGFP}^{+}$cells at the more 313 epicardial layers with the largest damaged area (Fig. 4f). Immunofluorescence analysis demonstrated 314 that the vast majority of $\mathrm{eGFP}^{+}$cells engrafted in the damaged tissue were $\mathrm{cTNT}^{+} \mathrm{CM}$ with elongated 315 shape, aligned myofibrils, and well-organized sarcomeres (Fig. 4g). Expression of the gap junction 316 protein connexin 43 was detected at the intercalated discs of $\mathrm{eGFP}^{+} \mathrm{CMs}$ and at the contact zone 317 between graft and host CMs (Fig. 4g). Moreover, immunostaining for the endothelial marker CD31 318 documented significantly enhanced neo-angiogenesis at the RFA site after HVP transplantation (Fig. $3194 \mathrm{~h}$ and Extended Data Fig. 7b). Notably, $\sim 6 \%$ of CD $31^{+}$cells were of human origin (Fig. 4h). No 320 evidence of acute graft rejection in the transgenic LEA29Y pigs under the immunosuppressive 321 regimen was observed on D14 post-transplantation, as assessed by CD68 immunodetection. 
322 Interestingly, we even observed a reduction of $\mathrm{CD}^{+} 8^{+}$cells at both injured and adjacent areas in HVP-

323 treated RFA compared to control (Extended Data Fig. 7c), suggesting that HVPs might directly

324 influence post-injury inflammation.

325 Ventricular arrhythmias have emerged as major side effect of CM cell therapy.

326 Electrophysiological studies in large animals consistently observed arrhythmias originating in graft

327 regions from ectopic pacemakers ${ }^{40,41}$. To assess the electrophysiological consequences of hPSC

328 derived HVPs, we subjected the two pigs euthanized on D14 to permanent ECG monitoring following

329 cell therapy and RFA injury using an implantable event recorder. No ventricular tachycardia (VT) was

330 observed before cell transplantation and only few non-sustained VT episodes occurred shortly within

331 the first $48 \mathrm{~h}$ of treatment (Fig. 4i).

\section{Discussion and future directions}

334 Our ex vivo chimeric model of human HVPs and NHP heart tissue provides an unprecedented system 335 to refine molecular pathways implicated in cardiac regeneration and healing at a single cell resolution.

336 As such it offers an innovative approach to predict outcome of cell-based regeneration with high

337 fidelity, which could be applied to other non-regenerative organs such as brain. We demonstrate that 338 HVPs harbour the unique potential to sense and counter-act injury by reactivating sequential 339 developmental programs for directed migration, fibroblast repulsion, and ultimate muscle 340 differentiation in the scar area (Fig. 4j).

341 scRNAseq data unravelled key signalling pathways underlying HVP-mediated heart repair and 342 scarless healing during an acute injury response, including SLIT2/ROBO1. It will be of particular 343 interest to evaluate whether pharmacological manipulation of such signalling pathway could 344 circumvent cell application. Moreover, future studies should assess whether HVP therapy could be 345 beneficial in clinical settings of chronic heart failure (e.g. ischemic heart disease, genetic 346 cardiomyopathies, and post-myocarditis) to reduce pre-existing fibrosis. Recently, the ESCORT trial 347 performed first transplants of hPSC-derived cardiac progenitors surgically delivered as patches onto 348 the heart's surface in patients with ischemic cardiomyopathy and reported no adverse side effects ${ }^{18}$. In 349 parallel, translational efforts have evaluated the potential of hPSC-derived CMs to engraft in the heart 350 of large animals, including primates. Electromechanical coupling and improvement of systolic LV 351 function have been reported ${ }^{17,40,41}$. However, concerns remain regarding the immaturity of engrafted 352 CMs, survival of the cells and the propensity for ventricular arrhythmias. We found that in vivo hPSC353 derived HVPs transplanted in the injured myocardium of LEA29Y transgenic pigs did not induce 
354 sustained VTs over a two week period. Yet, arrhythmogenic potential of cell grafts needs to be further

355 assessed in long-term analyses. We also observed an increased neovascularization in vivo, with a 356 proportion of endothelial cells being of human origin. Further studies need to demonstrate whether 357 such neovascularization response will be sufficient to restore normal blood flow. Robust arterial input 358 will be crucial for permanent functional improvement, which may require a combination of cell 359 therapy with other modalities.

360 In conclusion, our data indicate that HVPs harbour the unique capability to target both loss of 361 myocardium and fibrotic scarring in the primate heart, and support their therapeutic potential. 362 However, before HVP transplantation can be translated to humans much work remains to determine 363 whether pharmaceutical-grade batches of HVPs (purity $\geq 90 \%$, yields $\geq 10 \times 10^{7}$ cells) can be achieved, 364 safety risks related to ventricular arrhythmias can be excluded, and the use of hypo-immunogenic PSC 365 lines can circumvent long-term rejection. Developing innovative therapeutic strategies that are rooted 366 in fundamental biology of cardiac development could pave the way for successful cell-based cures of 367 heart disease. 


\section{Methods}

370 ESC maintenance, cardiac differentiation, and HVP MACS-based purification. Embryonic stem 371 cell lines ES03 NKX2.5 $5^{\mathrm{eGFP} / w t}$ and H9 NKX2.5 $5^{\mathrm{eGFP} / w t} 42$ were generously gifted from Dr. David Elliott, 372 MCRI Australia) and maintained on Matrigel-coated plates (BD Biosciences, Germany) in E8 373 medium (Gibco, USA) with daily medium change. After reaching a confluency of 85-90\%, cells were 374 passaged by dissociation into single cells using Accutase (Innovative Cell Technologies, USA) at $37537^{\circ} \mathrm{C}$ for 5 minutes and replated in a ratio of $1: 6$ or 1:9 on new Matrigel-coated plates in E8 376 supplemented with $5 \mu \mathrm{M}$ ROCK inhibitor Y-27632 (Stemcell Technologies, Canada) for $24 \mathrm{~h}$. 377 Differentiation to HVPs was achieved according to our previously published protocol ${ }^{28}$. Briefly, after 378 dissociation with Accutase, ESCs were plated on Matrigel-coated cell culture dishes at a density of $1 \times 10^{6} /$ well in E8 supplemented with $5 \mu \mathrm{M}$ ROCK for 24 hours followed by culture in E8. When full confluency was reached, differentiation was initiated on day -6 by adding RPMI/B27 minus insulin (Gibco, USA) supplemented with $1 \mu \mathrm{M}$ CHIR 98014 (Selleckchem, USA). Media was changed to RPMI/B27 minus insulin (HVP culture medium; CCM) after 24 hours. On day -3, a combined medium consisting of $1 \mathrm{ml}$ collected conditioned media and $1 \mathrm{ml}$ fresh CCM supplemented with $2 \mu \mathrm{M}$ Wnt-C59 (Selleckchem, USA) was applied and completely replaced by CCM on day -1. On day 0 HVPs were collected for MAC sorting. After dissociation into single cells with Accutase, cells were stained with Anti-TRA-1-60 MicroBeads (Miltenyi Biotec, Germany) before negative sorting with an autoMACS Pro Separator (Miltenyi Biotec, Germany) according to manufacturer's instructions. A fraction of the cells was stained with an anti-human TRA-1-60 antibody conjugated with Alexa Fluor 488 (StemCell Technologies, Canada) and analysed by flow cytometry using a BD FACSCantoII according to manufacturer's instructions. Batches with $<5 \%$ TRA-1-60 positive cells were used for in vivo transplantation experiments. To generate mature CMs, HVPs were cultured further in 12 well plates in RPMI/B27 containing insulin and medium was changed every other day. For long-term storage, cardiac progenitors (D0) or differentiated cardiomyocytes (D25) were frozen in CryoStor cell cryopreservation media (Sigma Aldrich, USA).

Ex vivo NHP heart slice culture. For ex vivo heart slice cultivation, freshly explanted NHP leftventricular myocardial tissue was placed in 2,3-Butanedione 2-Monoxime (BDM; Sigma Aldrich, USA) at $4^{\circ} \mathrm{C}$ and shipped from the German primate centre, Göttingen, where the primate had been euthanized in course of a vaccination study (file reference: 33.19-42502-04-16/2264), from Karolinska Institutet, Sweden (file reference: N 277/14) or from the Walter Brendel Institute, LMU, 400 Germany (file reference: ROB-55.2--2532.Vet_02-14-184). Within 12-24 hours, heart tissues were 
401 sectioned on vibratome (VT1200S, Leica Biosystems, Germany) to approximately $1 \mathrm{~cm} \times 2 \mathrm{~cm} \times 300$ $402 \mu \mathrm{m}$ thick tissue slices. Slices were anchored in biomimetic cultivation chambers (BMCC) via small 403 plastic triangles attached to the slices with tissue adhesive (Histoacryl; B. Braun, Germany) according 404 to fiber direction and subjected to physiological preload of $1 \mathrm{mN}$ and stimulation at $1 \mathrm{~Hz}(50 \mathrm{~mA}$ 405 pulse current, $1 \mathrm{~ms}$ pulse duration), as previously described ${ }^{29}$. The slices were maintained in M199 406 medium (Sigma Aldrich, USA) supplemented with 1\% Penicillin-Streptomycin, 0.5\% 407 Insulin/Transferrin/Selenium (Gibco, USA) and $50 \mu \mathrm{M}$ 2-Mercaptoethanol. Medium was replaced 408 every other day (2/3 fresh medium, 1/3 conditioned medium). The BMCCs were anchored on a rocker 409 plate, placed in an incubator at $37^{\circ} \mathrm{C}, 5 \% \mathrm{CO}_{2}, 20 \% \mathrm{O}_{2}$ and $80 \%$ humidity. Contractile force of the 410 constructs was continuously measured and contractility data were imported into and analysed by 411 LabChart Reader software (AD Instruments, New Zealand).

412 Generation of chimeric human-NHP heart constructs. Native NHP heart slices within BMCCs 413 were underpinned with a hand-trimmed filter $(0.40 \mu \mathrm{m}$ pore size $)$ and suspended in $0.5 \mathrm{ml} \mathrm{CCM}$ 414 underneath the filter. For homogenous cell seeding, $2 \times 10^{6}$ HVPs were thawed and immediately seeded 415 onto the tissue within a pluronic F-127 (concentration of $0.33 \mathrm{~g} / \mathrm{ml}$, Sigma Aldrich, USA) frame using 416 a bioprinting device (CANTER Bioprinter V4, Germany), equipped with a $0.58 \mathrm{~mm}$ standard Luer417 Lock nozzle (Vieweg® Dosiertechnik, Germany). For selective cell seeding, 0.5x10 ${ }^{6}$ HVPs or D25 $418 \mathrm{CMs}$ were used. In the first 12 hours after seeding, chimeric constructs were cultured in $500 \mu \mathrm{CCM}$ 419 supplemented with $5 \mu \mathrm{M}$ ROCK inhibitor underneath the filter within BMCCs without rocking and 420 electrical pacing. Twelve-24 hours after seeding, another $500 \mu \mathrm{l}$ CCM supplemented with $5 \mu \mathrm{M}$ 421 ROCK inhibitor were added under the filter and rocking (60 rpm, $15^{\circ}$ tilt angle) was resumed. After 422 24h, medium was entirely replaced with $1 \mathrm{ml} \mathrm{CCM}$. On day 2, co-culture slices were maintained in $4232.4 \mathrm{ml} \mathrm{CCM}$ with continuous electrical pacing $(1 \mathrm{~Hz})$ and rocking. Medium was replaced every other 424 day (2/3 fresh medium, 1/3 condition medium).

425 RFA-induced myocardial injury. Native NHP heart slices with selectively seeded HVPs or CMs 426 were injured on the opposite tissue border 3 days after cell seeding by applying $20 \mathrm{~W}$ for 15 sec using 427 a THERMOCOOL® SF Uni-Directional Catheter, tip electrode $3.5 \mathrm{~mm}$ (Biosense Webster, USA) and 428 Stockert 70 radiofrequency (RF) generator (Biosense Webster, USA). During the RFA procedure, 429 physiologic preload was reduced to $0.5 \mathrm{mN}$ within BMCC that was readjusted to $1 \mathrm{mN}$ after 2 days.

430 For in vivo experiments, epicardial radiofrequency ablation with $25 \mathrm{~W}$ for $7 \mathrm{sec}$ was performed to 431 produce a standardized, non-transmural injury. 
432 Proliferation analysis by flow cytometry. For quantification of proliferation, HVPs on day 0 of 433 monolayer culture and day 3, 7 and 14 of co-culture were incubated with $10 \mu \mathrm{M}$ EdU for 24 hours, 434 dissociated with $480 \mathrm{U} / \mathrm{ml}$ collagenase type II, fixed with 4\% paraformaldehyde (PFA) for 15 min at 435 room temperature (RT), washed three times with PBS and processed using the Click-iT EdU594 Flow 436 Cytometry Assay Kit (Thermo Fisher, USA) according to manufacturer's instructions. Flow 437 cytometry data were acquired with a Gallios flow cytometer (Beckman Coulter, USA) and evaluated 438 with Kaluza software version 1.2 (Beckman Coulter, USA).

$439 \mathbf{C a}^{2+}$ imaging of RFA-injured heart slices. NHP heart slices seeded with HVPs/CMs after RFA 440 injury were loaded with $3 \mu \mathrm{M}$ Fluo-4-AM in CCM (without phenol red) supplemented with $0.75 \%$ 441 Kolliphor EL (Sigma Aldrich, USA) by incubation at $37^{\circ} \mathrm{C}$ for 60 minutes, washed, and incubated for 442 another $30 \mathrm{~min}$ at $37^{\circ} \mathrm{C}$ to allow de-esterification of the dye in Tyrode's solution supplemented with $443 \mathrm{Ca}^{2+}\left(135 \mathrm{mM} \mathrm{NaCl}, 5.4 \mathrm{mM} \mathrm{KCl}, 1 \mathrm{mM} \mathrm{MgCl}, 10 \mathrm{mM}\right.$ glucose, $1.8 \mathrm{mM} \mathrm{CaCl}_{2}$, and $10 \mathrm{mM}$ 444 HEPES; pH7.35). Calcium signals from native NHP CMs and seeded HVPs were subsequently 445 imaged using an inverted microscope DMI6000 B (Leica, Germany) equipped with a 10x objective, a 446 GFP filter set and a Zyla V sCMOS camera (Andor Technology, UK). Point stimulation electrodes 447 were connected to an HSE stimulus generator (Hugo Sachs Elektronik, Germany) providing 448 depolarizing pulses (40 V, $3 \mathrm{~ms}$ duration) at $1 \mathrm{~Hz}$ on the tissue border opposite the RFA injury. 449 Imaging settings (illumination intensity, camera gain, binning) were adjusted to achieve an optimal 450 signal to noise ratio with an imaging rate of $14 \mathrm{~Hz}$. ImageJ ROI Manager was used to quantify 451 fluorescence over single cells and background regions. Subsequent analysis was performed in RStudio 452 using custom-written R scripts. After subtraction of background fluorescence, the time course of Fluo4534 signal intensity was expressed as arbitrary units.

454 Cell isolation for scRNA-sequencing. For scRNAseq, co-culture patches on day 0, 3 and 21 without 455 RFA, as well as on day 1 and 2 after RFA were dissociated using $20 \mathrm{U} / \mathrm{ml}$ papain ${ }^{43}$ (Worthington 456 Biochemical, USA), filtered through a $70 \mu \mathrm{m}$ filter and resuspended in 3\% BSA in PBS. Using 457 FACSAria III (Becton Dickinson), eGFP ${ }^{+}$cells on day 0,3 and 21 without RFA and eGFP ${ }^{+}$and eGFP $^{-}$ 458 cells on day 1 and 2 after RFA were sorted into individual wells of 384-well plates containing Smart459 Seq2 cell lysis buffer (ERCC 1:4 x10 dilution). The lysed single cells were stored at $-80^{\circ} \mathrm{C}$ prior to 460 complementary deoxyribonucleic acid (cDNA) synthesis, using the Smart-Seq2 protocol. The quality 461 of the cDNA was confirmed using Agilent Bioanalyzer (Agilent, USA) and RNAseq libraries were 462 prepared using in-house compatible Tn5 and Nextera index primers (Illumina, USA). Following a 463 final clean-up, the size distribution of the sequence libraries was verified using Agilent high- 
464 sensitivity chip and the concentration of each library was measured using the Qubit3 Fluorometer 465 (Thermo Fisher, USA).

466 scRNAseq and gene expression analysis. scRNAseq was performed at the sequencing facility of the 467 Karolinska Institutet using the Genome Analyzer HiSeq2500 (Illumina, USA) for single-end 468 sequencing of 56 bp. The Genome Analyzer Analysis Pipeline (Illumina, USA) was used to process 469 the sequencing files of raw reads in the FASTQ format. The cDNA insert was aligned to the 470 hg19/Mmul_1 reference genomes using Tophat2, combined with Bowtie2. Only confidently mapped 471 and non-PCR duplicates were used to generate the gene-barcode cell matrix. Further, quality check 472 steps, including the identification of highly variable genes, dimensionality reduction, standard 473 unsupervised clustering algorithms and the differentially expressed genes analysis were performed 474 using the standard Seurat R pipeline ${ }^{44}$.

475 Cell clustering, UMAP visualization and marker-gene identification. The gene-barcode matrix 476 was scaled, normalized and log-transformed. The dimensionality of the data was reduced by principal 477 component analysis (PCA) (20 components) first and then with UMAP (resolution $=0.3$ ). Then, all 478 cells from each cluster were sampled and differentially expressed genes across different clusters were 479 identified with the FindAllMarkers and FindMarkers functions of Seurat R package. Clusters were 480 assigned to known cell types on the basis of cluster-specific markers (Supplementary Table 1).

481 Integrated analysis of single-cell datasets. To integrate and validate the robustness of our analysis, 482 we took advantage of two recently published single cell profiles ${ }^{31,34}$. To reduce batch-effect 483 differences, we used the Seurat alignment re-scaling and re-normalizing for the integrated dataset. For 484 all new integrated datasets, we identified variable genes generating a new dimensional reduction that 485 was used for further analysis. Pseudotemporal ordering was done using Monocle $2^{45}$. In brief, an 486 integrated gene-expression matrix was constructed as described above. With the function 487 differentialGeneTest we analysed differentially expressed genes across different development 488 conditions. At max the top 3,000 genes with the lowest q value were used to construct the pseudotime 489 trajectory.

490 Determination of biological processes and molecular function on the basis of enrichment 491 analysis. Statistical analysis and visualization of gene sets were performed using the clusterProfiler R 492 package $^{46}$. GSEA databases were used to determine the enrichment of biological processes, cellular 493 components and molecular function on the basis of the genes that were significantly upregulated. 494 Process-specific signatures were defined by the top genes as ranked by the significance and 495 expression scores. 
NHP CF isolation and lentiviral transduction. Freshly explanted NHP left ventricular myocardium was minced into small pieces and incubated with $550 \mathrm{U} / \mathrm{ml}$ collagenase II (Worthington, USA) at $37^{\circ} \mathrm{C}$ for 15 minutes in a series of 6 digestions. Every 15 minutes the supernatant was collected and centrifuged at $300 \mathrm{~g}$ for 5 minutes and washed twice with DMEM/F-12 (Gibco, USA). Isolated cardiac fibroblasts (CFs) were cultured in CF Medium (CFM; DMEM-F12, 10\% fetal bovine serum, 2 $\mathrm{mM}$ L-Glutamine, $0.5 \%$ Penicillin/Streptomycin) at $37^{\circ} \mathrm{C}$ and $5 \% \mathrm{CO}_{2}$ with media change every other day. For CF passaging 0.05\% Trypsin-EDTA (Gibco, USA) was used.

For lentiviral transduction, a dsRed-expressing lentivirus was produced using a pRRLsin-18-PGK-d transfer plasmid combined with the packaging plasmid (pCMVdR8.74) and the envelope plasmid (pMD2.VSV.G) in HEK293T cells. The CFs were incubated with PGK-dsRed lentivirus and $8 \mu \mathrm{g} / \mathrm{mL}$ of polybrene hexadimethrine bromide (Sigma Aldrich, USA) for 24 hours at $37^{\circ} \mathrm{C}, 5 \% \mathrm{CO}_{2}$ and the transduction efficiency was evaluated by dsRed expression after 96 hours.

Monolayer co-culture of HVPs and CFs for cell interaction studies after RFA injury. NHP$\mathrm{CF}^{\mathrm{dsRed}}\left(1 \times 10^{4} /\right.$ well) were seeded in 4-well chamber slides (Thermo Fisher, USA) coated with fibronectin (Sigma Aldrich, USA). After 3 days, RFA injury (20 W, 7 sec) was introduced on one border of the chamber slide followed by seeding of $5 \times 10^{5}$ HVPs on the opposite side. Cellular migration and interaction were studied by time-lapse microscopy (image acquisition every 90 minutes for 3 days; daily medium change with CCM). For the analysis of CF repulsion, anti-ROBO1 (5 $\mu \mathrm{g} / \mathrm{ml})$ and rhSLIT2 $(2 \mu \mathrm{g} / \mathrm{ml})$ treatments (Supplementary Table 2) were performed on days 7 and 8 after RFA injury and HVP seeding and stopped after 10 minutes, 40 minutes and 24 hours. Video clips were analysed with ImageJ for cell movement of HVPs and CFs by TrackMate plug-in ${ }^{47}$.

Identification of migratory signaling by trans-well migration assay. For trans-well migration studies, the CytoSelectTM Cell Migration and Invasion Assay (Cell Biolabs, USA) was used and samples were processed according to manufacturer's instructions. In brief, $0.5 \times 10^{6}$ HVPs (D0) were suspended in $300 \mu \mathrm{l}$ serum-free medium and plated on the upper compartment of the trans-well migration assay (polycarbonate membrane inserts; $8 \mu \mathrm{m}$ pore size). The chemoattractant factor CXCL12 was added in two different concentrations (low dose $=20 \mathrm{ng} / \mathrm{ml}$, high dose $=80 \mathrm{ng} / \mathrm{ml}$ ) to $500 \mu 1$ RPMI in the lower compartment. Agents that inhibit cell migration were added directly to the cell suspension in the upper compartment (CXCR4-RB (12 $\mu \mathrm{g} / \mathrm{ml})$, SDC4-RB (1:500), ITGB1-RB (8 $\mu \mathrm{g} / \mathrm{ml})$, ACKR-RB (10 $\mu \mathrm{g} / \mathrm{ml})$ ) (Supplementary Table 2). The cells were incubated for 24 hours in a

526 standard cell culture incubator. For quantification of migratory cells, medium was carefully aspirated 527 from the upper compartment and all non-migratory cells inside the insert were removed with cotton- 
528 tipped swabs. Next, inserts were stained in $400 \mu \mathrm{l}$ Cell Stain Solution for 10 minutes at RT, followed

529 by $5 \mathrm{x}$ washing with PBS and air-drying. Migratory cells were imaged with a light microscope under

530 100x magnification objective, with at least three individual fields per insert.

531 Label-free secretome analysis. Protease inhibitors (Complete mini EDTA Free, Thermo Fisher, 532 USA) were added to collected medium and supernatant concentration was achieved by ultrafiltration 533 using MWCO protein concentrators (Thermo Fisher, USA). Proteins (50 $\mu \mathrm{g}$ ) were diluted in 1\% SDS, $53450 \mathrm{mM}$ dithiothreitol, $100 \mathrm{mM}$ Tris buffer ( $\mathrm{pH} \mathrm{8.0)}$ ), denatured for $10 \mathrm{~min}$ at $95^{\circ} \mathrm{C}$ and digested by 535 filter-aided (Merck Millipore, USA) sample preparation for proteome analysis (FASP) ${ }^{48}$. Next, 536 overnight digestion was performed at $37^{\circ} \mathrm{C}$ with $60 \mu$ digest buffer containing $500 \mathrm{ng}$ trypsin (Sigma 537 Aldrich, USA) in $50 \mathrm{mM}$ triethylammonium bicarbonate buffer ( $\mathrm{pH} \mathrm{8.5).} \mathrm{Peptides} \mathrm{were} \mathrm{recovered} \mathrm{by}$ 538 adding $140 \mu \mathrm{l}$ HPLC-grade water and centrifugation of filters for 25 minutes at 14,000 g (final 539 volume: $200 \mu \mathrm{l}$ tryptic digest). An aliquot corresponding to $10 \%$ of the digest volume was purified by 540 strong cation exchange StageTips ${ }^{49}$. The eluate $(10 \mu$ containing $500 \mathrm{mM}$ ammonium acetate in $20 \%$ 541 acetonitrile) was diluted $10 \mathrm{x}$ in $0.2 \%$ formic acid and injected for nanoscale LC-MS/MS analysis (4 $542 \mu \mathrm{l})$. Tryptic peptides were analysed by nanoscale LC-MS/MS by a Top-12 data-dependent analysis method run on a Q-Exactive "classic" instrument (Thermo Fisher, USA) ${ }^{50}$ with a gradient length of 85

544 min. Raw data were loaded in MaxQuant (v 1.6.2.6a) for database search and label-free quantification 545 by the MaxLFQ algorithm (Cox Mann, MCP 2014). For data processing, default parameters in MaxQuant were adopted, except for the following: LFQ min. ratio count: 2; fast LFQ: ON; quantification on unique peptides; match between runs (MBR): activated between technical replicates, not between different samples (achieved by assigning a separate sample group to each biological sample and allowing MBR each group only). For protein identification, MS/MS data were queried using the Andromeda search engine implemented in MaxQuant against the Homo Sapiens Reference Proteome (accessed in December 2019, 74,788 sequences) and the Macaca Fascicularis Reference

552 Proteome (accessed in January 2020, 46,259 sequences).

PCR). Total RNA of CFs/HVPs co-culture and conditioned CFs exposed to co-culture medium was extracted using the Absolutely RNA Miniprep Kit (Agilent Technologies, USA) according to the manufacturer's instructions and $1 \mu \mathrm{g}$ was reverse transcribed using the High-Capacity cDNA Reverse

557 Transcription kit (Thermo Fisher, USA). qRT-PCR was performed using $25 \mathrm{ng}$ cDNA per reaction 558 and the Power SYBR Green PCR Master Mix (Thermo Fisher, USA). Gene expression levels were assessed in three independent biological samples and normalized to glyceraldehyde-3-phosphate 
560 dehydrogenase (GAPDH) expression by using the comparative cycles of threshold $(\mathrm{Ct})$ method $(\triangle \mathrm{Ct})$. qRT-PCR assays were run on a 7500 Fast Real Time PCR system (Thermo Fisher, USA) and the data were processed using 7500 software v2.3. Following primers were used: GAPDH_Fw: TCCTCTGACTTCAACAGCGA; GAPDH_Rv: GGGTCTTACTCCTTGGAGGC; ROBO1_Fw: GGGGGAGAGAGAGTGGAGAC; ROBO1_Rv：AGGCTCTCCTACTGCAACCA; SLIT2_Fw: TAGTGCTGGCGATCCTGAA; SLIT2_Rv: GCTCCTCTTTCAATGGTGCT.

Pig experiments and treatments. Pigs were sedated by intramuscular injection of ketamine (Ursotamin $^{\circledR}$, Serumwerk Bernburg, Germany), azaperone (Stresnil ${ }^{\circledR}$, Elanco Animal Health, Bad Homburg, Germany) and atropinsulfate (B. Braun, Melsungen, Germany) and kept in full anaesthesia with mechanical ventilation by continuous intravenous application of propofol (Narcofol ${ }^{\circledR}, \mathrm{CP}$ Pharma, Burgdorf, Germany) and fentanyl (Fentadon ${ }^{\circledR}$, Eurovet Animal Health BV, Bladel, Netherlands). Following left lateral thoracotomy, the pericardium was opened and the anterior wall of the left ventricle was exposed. After induction of RFA injuries and injection of HVPs, the thorax was closed using multiple layers of sutures. To continuously monitor heart rate and function during the follow-up period, a cardiac event recorder (BioMonitor 2, Biotronik, Berlin, Germany) was implanted subcutaneously on the left thorax wall of the D14 group pigs. All pigs had a central venous catheter (Careflow ${ }^{\mathrm{TM}}$, Merit Medical, Galway, Ireland) inserted via the lateral ear vein that remained in place over the whole course of the follow up period.

For additional immunosuppression, $5 \mathrm{mg} / \mathrm{kg}$ methylprednisolone was applied intravenously on day 1 and $2.5 \mathrm{mg} / \mathrm{kg}$ on day 2 (Urbason ${ }^{\circledR}$, Sanofi-Aventis, Frankfurt, Germany). Further, all pigs received a once daily oral dose of $0.2 \mathrm{mg} / \mathrm{kg}$ tacrolimus (Prograf ${ }^{\circledR}$, Astellas Pharma, Munich, Germany) and $20 \mathrm{mg} / \mathrm{kg}$ oral mycophenolat-mofetil (CellCept ${ }^{\circledR}$, Roche, Penzberg, Germany) twice daily over the whole course of the experiment. For euthanasia, pigs were placed in full anaesthesia as described above and circulation was terminated by systemic injection of potassium chloride (B. Braun, Melsungen, Germany).

All pig experiments were performed with permission of the local regulatory authority, Regierung von were reviewed by the ethics committee according to $\S 15$ TSchG German Animal Welfare Law. cells were used for transplantation experiments in pigs. Briefly, $6 \times 10^{7}$ sorted HVPs were suspended in $10 \mu \mathrm{l} \mathrm{CCM} \mathrm{supplemented} \mathrm{with} 5 \mu \mathrm{M}$ ROCK inhibitor on the day of transplantation. Cells were spun down after thawing, and only the cell pellet was transplanted using insulin syringe. After exposing the 
592 pig heart by lateral thoracotomy and generation of non-transmural RFA injury as described above, the

593 cell suspension was injected approximately $1 \mathrm{~cm}$ apart from the injury. At injection sides, U-stitches

594 with pericardial patches were placed and closed using a tourniquet to reduce cell loss during cell

595 application (Extended Data Movie 2).

596 Sample processing and immunofluorescence analysis. Cells on chamber slides were fixed with 4\%

597 PFA for $10 \mathrm{~min}$ at RT. Co-culture 3D constructs were fixed in $4 \%$ PFA for $30 \mathrm{~min}$ at $4^{\circ} \mathrm{C}$, 598 cryopreserved with ice-cold methanol/acetone for $10 \mathrm{~min}$ at $-20^{\circ} \mathrm{C}$ and sectioned at $12 \mu \mathrm{m}$ in Tissue599 Tek O.C.T. compound (Sakura Finetek, JP). Freshly explanted pig hearts on D3, D5 and D14 after 600 RFA injury and HVP injection were examined for macroscopic signs of tumour formation before 601 RFA-injured areas with corresponding HVP/control injection sites were manually excised and fixed 602 with $4 \%$ PFA for 24 hours at $4^{\circ} \mathrm{C}$, followed by cryopreservation with ice-cold methanol/acetone for $60330 \mathrm{~min}$ at $-20^{\circ} \mathrm{C}$ and sectioning at $12 \mu \mathrm{m}$ in O.C.T. compound.

604 For immunofluorescence staining, samples were washed three times with PBS, permeabilised and 605 blocked with $0.1 \%$ Triton X-100 and 10\% fetal bovine serum (FBS) for 2 hours at RT (co-culture and 606 monolayer samples) or overnight at $4^{\circ} \mathrm{C}$ (in vivo samples).

607 Primary antibodies against desired epitopes (Supplementary Table 2) were incubated overnight at $4^{\circ} \mathrm{C}$ 608 at the indicated dilutions in 1\% FBS, 0.1\% Triton X-100 in PBS. After washing five times with $0.1 \%$ 609 Triton X-100 in PBS for 5 minutes, appropriate secondary antibodies (1:500) were added in 1\% FBS, $6100.1 \%$ Triton X-100 in PBS for 2 hours at RT protected from light. After washing three times for 5 611 minutes with $0.1 \%$ Triton X-100 in PBS, Hoechst 33258 was added at a final concentration of 5 $612 \mu \mathrm{g} / \mathrm{mL}$ in PBS for 15 minutes at RT. After washing three times with PBS, samples were covered with 613 fluorescence mounting medium (Dako, USA) and stored at $4^{\circ} \mathrm{C}$. Images were acquired using a 614 DMI6000-AF6000 or SP8 confocal laser-scanning Leica microscope. Images were assigned with 615 pseudo-colors and processed with ImageJ. Quantification of scar volumes, eGFP ${ }^{+}$area and cell-type 616 proportions were performed with image $\mathrm{J}$ cell counter notice and volume calculator.

617 Statistical analysis. Statistical analyses - excluding scRNAseq experiments - are presented as 618 mean \pm SEM unless otherwise indicated. Two groups were compared using an unpaired $t$-test. 619 Statistical tests were performed on the online platform socscstatistics.com. Significance was defined 620 as $* \mathrm{p} \leq 0.05, * * \mathrm{p} \leq 0.01, * * * \mathrm{p} \leq 0.001$.

621 Data and CODE availability. The RNAseq data generated during this study are available at the GEO 622 database with project number GEO: GSE153282. The mass spectrometry data have been deposited to 623 the ProteomeXchange Consortium via the PRIDE database with the dataset identifier PXD019521. 


\section{References}

1 Lozano, R. et al. Global and regional mortality from 235 causes of death for 20 age groups in 1990 and 2010: a systematic analysis for the Global Burden of Disease Study 2010. Lancet 380, 2095-2128, doi:10.1016/S0140-6736(12)61728-0 (2012).

2 Tzahor, E. \& Poss, K. D. Cardiac regeneration strategies: Staying young at heart. Science 356, 1035-1039, doi:10.1126/science.aam5894 (2017).

3 Bergmann, O. et al. Dynamics of Cell Generation and Turnover in the Human Heart. Cell 161, 1566-1575, doi:10.1016/j.cell.2015.05.026 (2015).

4 Papait, R. et al. Histone Methyltransferase G9a Is Required for Cardiomyocyte Homeostasis and Hypertrophy. Circulation 136, 1233-1246, doi:10.1161/CIRCULATIONAHA.117.028561 (2017).

5 Travers, J. G., Kamal, F. A., Robbins, J., Yutzey, K. E. \& Blaxall, B. C. Cardiac Fibrosis: The Fibroblast Awakens. Circ Res 118, 1021-1040, doi:10.1161/CIRCRESAHA.115.306565 (2016).

6 Fang, L., Murphy, A. J. \& Dart, A. M. A Clinical Perspective of Anti-Fibrotic Therapies for Cardiovascular Disease. Front Pharmacol 8, 186, doi:10.3389/fphar.2017.00186 (2017).

$7 \quad$ Porrello, E. R. et al. Transient regenerative potential of the neonatal mouse heart. Science 331, 1078-1080, doi:10.1126/science. 1200708 (2011).

8 Hnatiuk, A. \& Mercola, M. Stars in the Night Sky: iPSC-Cardiomyocytes Return the Patient Context to Drug Screening. Cell Stem Cell 24, 506-507, doi:10.1016/j.stem.2019.03.013 (2019).

9 Desgres, M. \& Menasche, P. Clinical Translation of Pluripotent Stem Cell Therapies: Challenges and Considerations. Cell Stem Cell 25, 594-606, doi:10.1016/j.stem.2019.10.001 (2019).

10 Shi, Y., Inoue, H., Wu, J. C. \& Yamanaka, S. Induced pluripotent stem cell technology: a decade of progress. Nat. Rev. Drug Discov. 16, 115-130, doi:10.1038/nrd.2016.245 (2017).

11 Smart, N. et al. De novo cardiomyocytes from within the activated adult heart after injury. Nature 474, 640-644, doi:10.1038/nature10188 (2011).

12 Gabisonia, K. et al. MicroRNA therapy stimulates uncontrolled cardiac repair after myocardial infarction in pigs. Nature 569, 418-422, doi:10.1038/s41586-019-1191-6 (2019).

13 Wei, K. et al. Epicardial FSTL1 reconstitution regenerates the adult mammalian heart. Nature 525, 479-485, doi:10.1038/nature15372 (2015).

14 Bassat, E. et al. The extracellular matrix protein agrin promotes heart regeneration in mice. Nature 547, 179-184, doi:10.1038/nature22978 (2017).

15 Monroe, T. O. et al. YAP Partially Reprograms Chromatin Accessibility to Directly Induce Adult Cardiogenesis In Vivo. Dev. Cell 48, 765-779 e767, doi:10.1016/j.devcel.2019.01.017 (2019).

16 Srivastava, D. \& DeWitt, N. In Vivo Cellular Reprogramming: The Next Generation. Cell 166, 1386-1396, doi:10.1016/j.cell.2016.08.055 (2016).

17 Chong, J. J. et al. Human embryonic-stem-cell-derived cardiomyocytes regenerate non-human primate hearts. Nature 510, 273-277, doi:10.1038/nature13233 (2014).

18 Menasche, P. et al. Transplantation of Human Embryonic Stem Cell-Derived Cardiovascular Progenitors for Severe Ischemic Left Ventricular Dysfunction. J Am Coll Cardiol 71, 429-438, doi:10.1016/j.jacc.2017.11.047 (2018).

19 Chien, K. R. et al. Regenerating the field of cardiovascular cell therapy. Nat Biotechnol 37, 232-237, doi:10.1038/s41587-019-0042-1 (2019). 
20 Sadek, H. \& Olson, E. N. Toward the Goal of Human Heart Regeneration. Cell Stem Cell 26, 7-16, doi:10.1016/j.stem.2019.12.004 (2020).

21 Vagnozzi, R. J. et al. An acute immune response underlies the benefit of cardiac stem cell therapy. Nature 577, 405-409, doi:10.1038/s41586-019-1802-2 (2020).

22 Eschenhagen, T. et al. Cardiomyocyte Regeneration: A Consensus Statement. Circulation 136, 680-686, doi:10.1161/CIRCULATIONAHA.117.029343 (2017).

23 Brade, T., Pane, L. S., Moretti, A., Chien, K. R. \& Laugwitz, K. L. Embryonic heart progenitors and cardiogenesis. Cold Spring Harbor perspectives in medicine 3, a013847, doi:10.1101/cshperspect.a013847 (2013).

24 Sahara, M., Santoro, F. \& Chien, K. R. Programming and reprogramming a human heart cell. EMBO J 34, 710-738, doi:10.15252/embj.201490563 (2015).

25 Moretti, A. et al. Multipotent embryonic isl1+ progenitor cells lead to cardiac, smooth muscle, and endothelial cell diversification. Cell 127, 1151-1165 (2006).

26 Spater, D. et al. A HCN4+ cardiomyogenic progenitor derived from the first heart field and human pluripotent stem cells. Nat Cell Biol 15, 1098-1106, doi:10.1038/ncb2824 (2013).

27 Liang, X. et al. HCN4 dynamically marks the first heart field and conduction system precursors. Circulation Research 113, 399-407, doi:10.1161/CIRCRESAHA.113.301588 (2013).

28 Foo, K. S. et al. Human ISL1(+) Ventricular Progenitors Self-Assemble into an In Vivo Functional Heart Patch and Preserve Cardiac Function Post Infarction. Mol Ther 26, 16441659, doi:10.1016/j.ymthe.2018.02.012 (2018).

29 Fischer, C. et al. Long-term functional and structural preservation of precision-cut human myocardium under continuous electromechanical stimulation in vitro. Nat Commun 10, 117, doi:10.1038/s41467-018-08003-1 (2019).

$30 \mathrm{Bu}$, L. et al. Human ISL1 heart progenitors generate diverse multipotent cardiovascular cell lineages. Nature 460, 113-117 (2009).

31 Sahara, M. et al. Population and Single-Cell Analysis of Human Cardiogenesis Reveals Unique LGR5 Ventricular Progenitors in Embryonic Outflow Tract. Dev. Cell 48, 475-490 e477, doi:10.1016/j.devcel.2019.01.005 (2019).

32 Lescroart, F. et al. Defining the earliest step of cardiovascular lineage segregation by singlecell RNA-seq. Science 359, 1177-1181, doi:10.1126/science.aao4174 (2018).

33 Trapnell, C. et al. The dynamics and regulators of cell fate decisions are revealed by pseudotemporal ordering of single cells. Nat Biotechnol 32, 381-386, doi:10.1038/nbt.2859 (2014).

34 Wang, L. et al. Single-cell reconstruction of the adult human heart during heart failure and recovery reveals the cellular landscape underlying cardiac function. Nat Cell Biol 22, 108-119, doi:10.1038/s41556-019-0446-7 (2020).

35 Brule, S. et al. The shedding of syndecan-4 and syndecan-1 from HeLa cells and human primary macrophages is accelerated by SDF-1/CXCL12 and mediated by the matrix metalloproteinase-9. Glycobiology 16, 488-501, doi:10.1093/glycob/cwj098 (2006).

36 Xiong, H. et al. Single-Cell Transcriptomics Reveals Chemotaxis-Mediated Intraorgan Crosstalk During Cardiogenesis. Circ Res 125, 398-410, doi:10.1161/CIRCRESAHA.119.315243 (2019).

37 Gonzalez-Rosa, J. M., Martin, V., Peralta, M., Torres, M. \& Mercader, N. Extensive scar formation and regression during heart regeneration after cryoinjury in zebrafish. Development 138, 1663-1674, doi:10.1242/dev.060897 (2011).

38 Nguyen Ba-Charvet, K. T. et al. Diversity and specificity of actions of Slit2 proteolytic fragments in axon guidance. $J$ Neurosci 21, 4281-4289 (2001). 
Bahr, A. et al. Ubiquitous LEA29Y Expression Blocks T Cell Co-Stimulation but Permits

40 Shiba, Y. et al. Allogeneic transplantation of iPS cell-derived cardiomyocytes regenerates Sexual Reproduction in Genetically

primate hearts. Nature 538, 388-391, doi:10.1038/nature19815 (2016).

41 Romagnuolo, R. et al. Human Embryonic Stem Cell-Derived Cardiomyocytes Regenerate the Infarcted Pig Heart but Induce Ventricular Tachyarrhythmias. Stem Cell Reports 12, 967-981, doi:10.1016/j.stemcr.2019.04.005 (2019).

42 Elliott, D. A. et al. NKX2-5(eGFP/w) hESCs for isolation of human cardiac progenitors and cardiomyocytes. Nat Methods 8, 1037-1040, doi:10.1038/nmeth.1740 (2011).

43 Fischer, B. et al. A complete workflow for the differentiation and the dissociation of hiPSCderived cardiospheres. Stem Cell Res 32, 65-72, doi:10.1016/j.scr.2018.08.015 (2018).

44 Satija, R., Farrell, J. A., Gennert, D., Schier, A. F. \& Regev, A. Spatial reconstruction of single-cell gene expression data. Nat. Biotechnol. 33, 495-502, doi:10.1038/nbt.3192 (2015).

45 Qiu, X. et al. Single-cell mRNA quantification and differential analysis with Census. Nat Methods 14, 309-315, doi:10.1038/nmeth.4150 (2017).

46 Yu, G., Wang, L. G., Han, Y. \& He, Q. Y. clusterProfiler: an R package for comparing biological themes among gene clusters. OMICS 16, 284-287, doi:10.1089/omi.2011.0118 (2012).

47 Tinevez, J. Y. et al. TrackMate: An open and extensible platform for single-particle tracking. Methods 115, 80-90, doi:10.1016/j.ymeth.2016.09.016 (2017).

48 Rappsilber, J., Mann, M. \& Ishihama, Y. Protocol for micro-purification, enrichment, prefractionation and storage of peptides for proteomics using StageTips. Nat Protoc 2, 18961906, doi:10.1038/nprot.2007.261 (2007).

49 Rappsilber, J. \& Mann, M. Analysis of the topology of protein complexes using cross-linking and mass spectrometry. CSH Protoc 2007, pdb prot4594, doi:10.1101/pdb.prot4594 (2007).

50 Laria, A. E. et al. Secretome Analysis of Hypoxia-Induced 3T3-L1 Adipocytes Uncovers Novel Proteins Potentially Involved in Obesity. Proteomics 18, e1700260, doi:10.1002/pmic.201700260 (2018). 


\section{Acknowledgements}

753 We thank Drs. Rabea Hinkel (Deutsches Primatenzentrum Göttingen), Mats Spångberg, Bengt 754 Eriksson, Astrid Fagreaus, and Pia Ekeland (Karoliska Institute), as well as Jan-Michael Abicht and 755 Matthias Längin (Walter Brendel Centre of Experimental Medicine, LMU) for providing NHP hearts, 756 Dr. David Elliott for his generosity of the two NKX2.5-eGFP cell lines, Dr. Alexander Goedel, Dr. 757 Ran Yang, and Dr. Xidan Li for FACS and bioinformatics assistance, and Dr. Steffen Dietzel (Core 758 Facility Bioimaging, Biomedical Center, LMU) for 2-Photon live microscopy. We would like to 759 acknowledge Birgit Campbell and Christina Scherb for their technical assistance in cell culture and 760 Sarah Luger (moments-of-aha.com) for graphic illustrations. This work was supported by grants from: 761 the European Research Council, ERC 743225 (to K.R.C.) and ERC 788381 (to A.M.); the German 762 Research Foundation, Transregio Research Unit 152 (to A.M., K-L.L.) and 267 (to A.M., K-L.L., and 763 C.K.); Swedish Research Council Distinguish Professor Grant (to K.R.C.); DZHK (German Centre for 764 Cardiovascular Research).

\section{Author contributions}

767 K-L.L, A.Mo., and K.R.C. together setup the collaboration and conceived the overall experimental plan. C.S. and M.T.D.A. performed functional experiments and histochemistry, analysed data, and generated figures. K.S.F. produced HVPs and CMs, performed MACS sorting, and analysed data. C.S., M.T.D.A., and K.S.F. contributed to the conception and design of experiments. G.S. performed bioinformatics analyses. F.R. and P.H. established RFA and measured scar parameters. T.D. conducted FACS analysis. T.D., I.M., and A.Me. produced heart slices and performed cellular seeding. Y.L.T. contributed to bioinformatics. S.S. developed cellular bioprinting. K.L., R.T., and A.D. introduced and adapted biomimetic slice culture. D.S. conducted calcium imaging and analysis and analysed time-lapse. E.P., M.G., and G.C. executed mass spectrometry. A.B., N.H., M.K., and C.K. performed in vivo pig experiments. M.K. injected HVPs in vivo. V.J. performed CD68 immunodetection and analysis. N.K. generated and provided transgenic LEA29Y pigs. N.K., C.K. and A.B. supervised in vivo studies and provided conceptual advice. A.Mo., K.R.C., and K.-L.L. conceived and supervised this study and provided financial support. A.Mo., K.R.C., and K.-L.L. wrote 
bioRxiv preprint doi: https://doi.org/10.1101/2020.07.03.183798; this version posted July 4 , 2020. The copyright holder for this preprint (which

was not certified by peer review) is the author/funder, who has granted bioRxiv a license to display the preprint in perpetuity. It is made available under aCC-BY-NC-ND 4.0 International license.

\section{Competing interests statement}

784 The authors declare that they have no competing financial interests. K.S.F. and K.R.C. are co785 inventors on a patent based on the HVP technology and its applications. The HVP intellectual

786 property is assigned to SWIBCO, a Swedish holding company. 
a
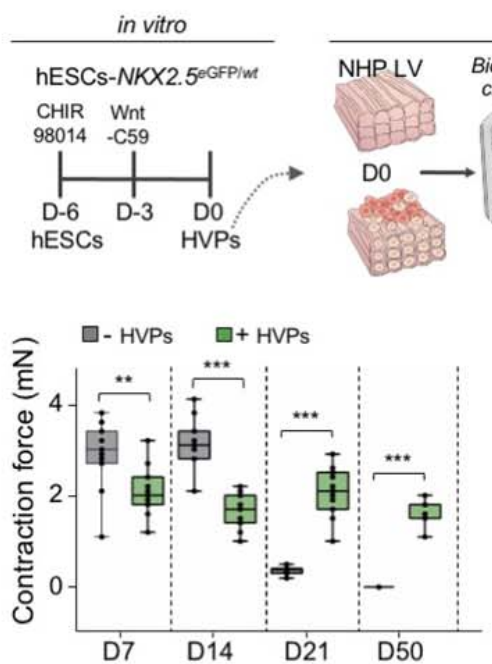

d

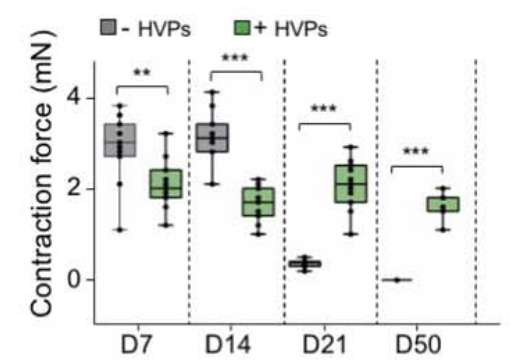

C

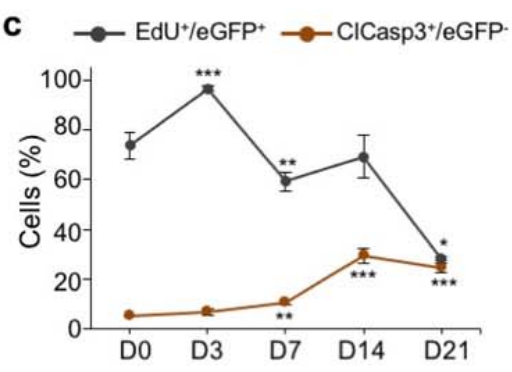

a-GFP MLC2v MLC2a DNA

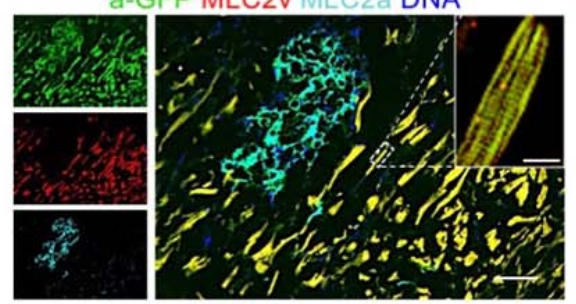

e

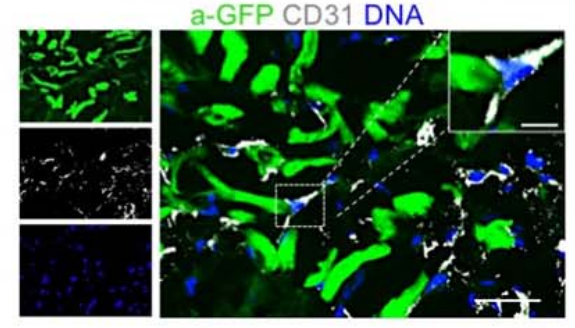

ex vivo

2

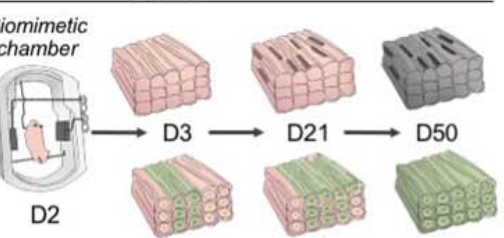

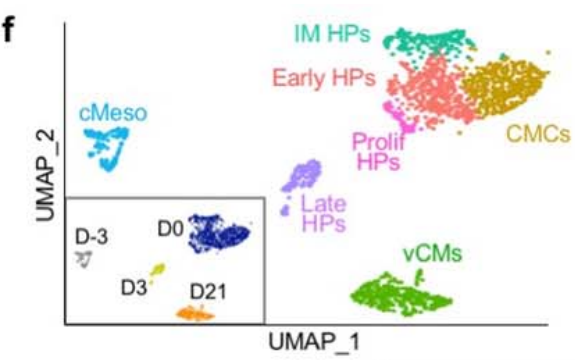

g

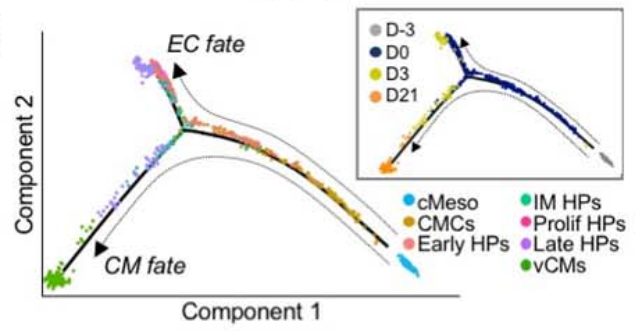

h

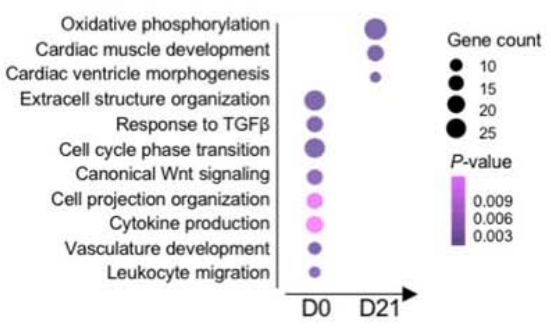

i

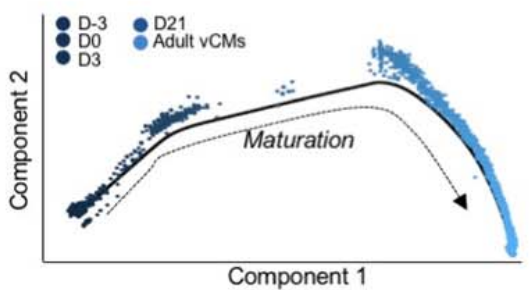

Figure 1. HVPs expand, repopulate and functionally mature in an ex vivo 3D NHP heart hESCs (left) and their ex vivo co-culture with native NHP LV slices in biomimetic chambers (right). b, Contractile force of ex vivo cultured NHP heart slices with and without HVPs on indicated days (D) of co-culture. Box plot shows all data points as well as the median and quartiles; $\mathrm{n}=11$ patches/time point; ${ }^{* *} \mathrm{p}<0.005,{ }^{* * *} \mathrm{p}<0.001$ ( $t$-test). c, Percentage of $\mathrm{EdU}^{+} / \mathrm{eGFP}^{+}$and $\mathrm{ClCasp}^{+} / \mathrm{eGFP}^{-}$cells during co-culture. Data are mean $\pm \mathrm{SEM} ; \mathrm{n} \geq 3$ samples/time point; ${ }^{*} \mathrm{p}<0.05,{ }^{* *} \mathrm{p}<0.005,{ }^{* * *} \mathrm{p}<0.001$ vs D0 ( $t$-test). d, e, Left, representative immunofluorescence images of D50 chimeric human-NHP heart constructs using an antibody against GFP (a-GFP) together with antibodies for MLC2a and MLC2v (d) or CD31 (e). Scale bar $100 \mu \mathrm{m}$ in d, $50 \mu \mathrm{m}$ in e, $10 \mu \mathrm{m}$ in insets. Right, percentage of eGFP ${ }^{+}$cells expressing MLC2v, MLC2a, or both (d) and human cells expressing CD31 (e) on D21 and D50. $\mathrm{HuNu}$, human nuclear antigen. Data are mean $\pm \mathrm{SEM} ; \mathrm{n}=4$ samples/time point; ${ }^{* *} \mathrm{p}<0.005$, $* * * p<0.001$ ( $t$-test). f, UMAP clustering of single cells captured on D-3 and D0 of in vitro differentiation together with D3 and D21 of ex vivo co-culture. cMeso, cardiac mesoderm; CMCs, cardiac mesenchymal cells; Early HPs, early heart progenitors; IM HPs, intermediate heart progenitors; Late HPs, late heart progenitors; vCMs, ventricular cardiomyocytes. g, Developmental 
bioRxiv preprint doi: https://doi.org/10.1101/2020.07.03.183798; this version posted July 4, 2020. The copyright holder for this preprint (which was not certified by peer review) is the author/funder, who has granted bioRxiv a license to display the preprint in perpetuity. It is made available under aCC-BY-NC-ND 4.0 International license.

807 endothelial cell. h, Representative GO terms upregulated during ex vivo co-culture. i, Pseudotime 808 trajectory of captured cells combined with adult vCMs from Wang et al. 2020. Colour gradient (from 809 dark to light) according to maturation.

810 

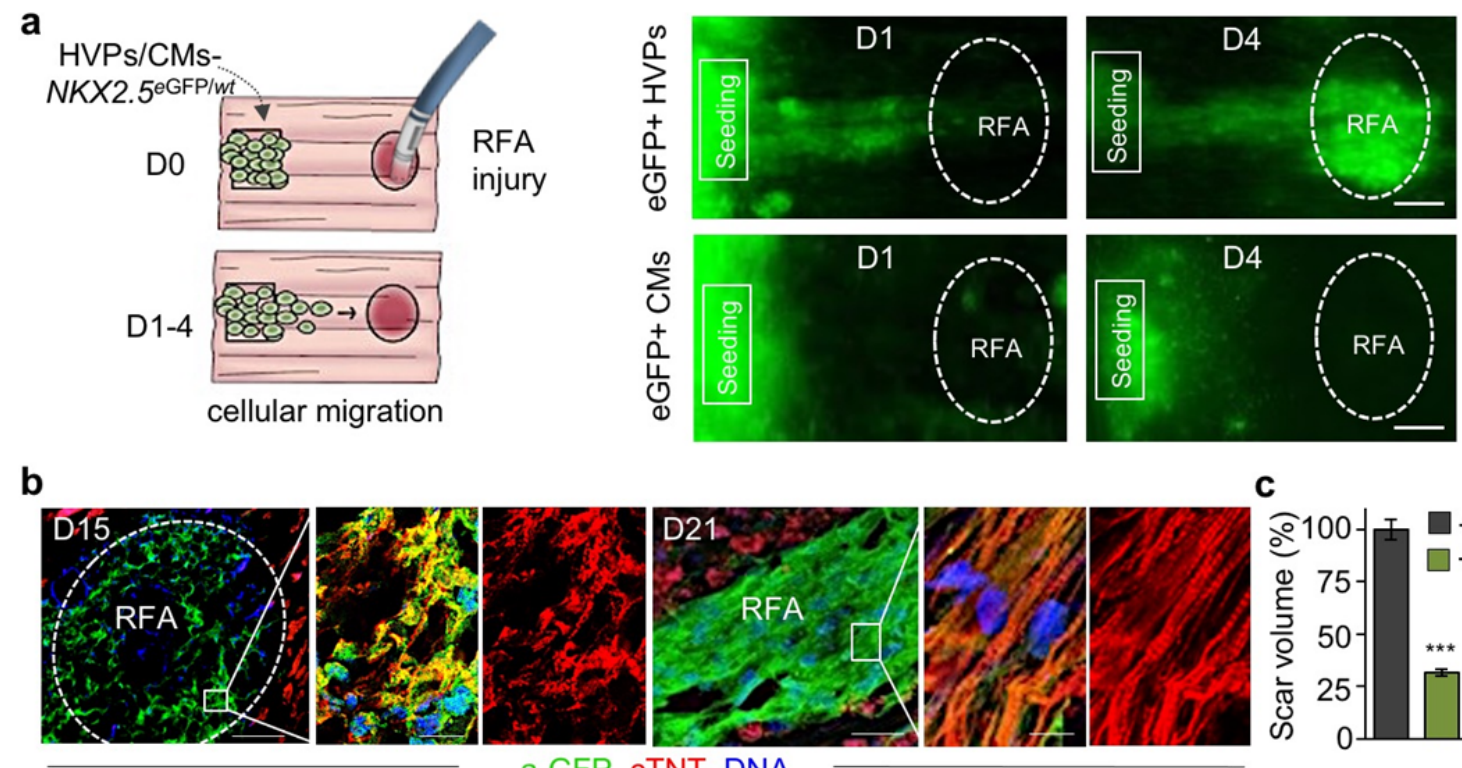

d
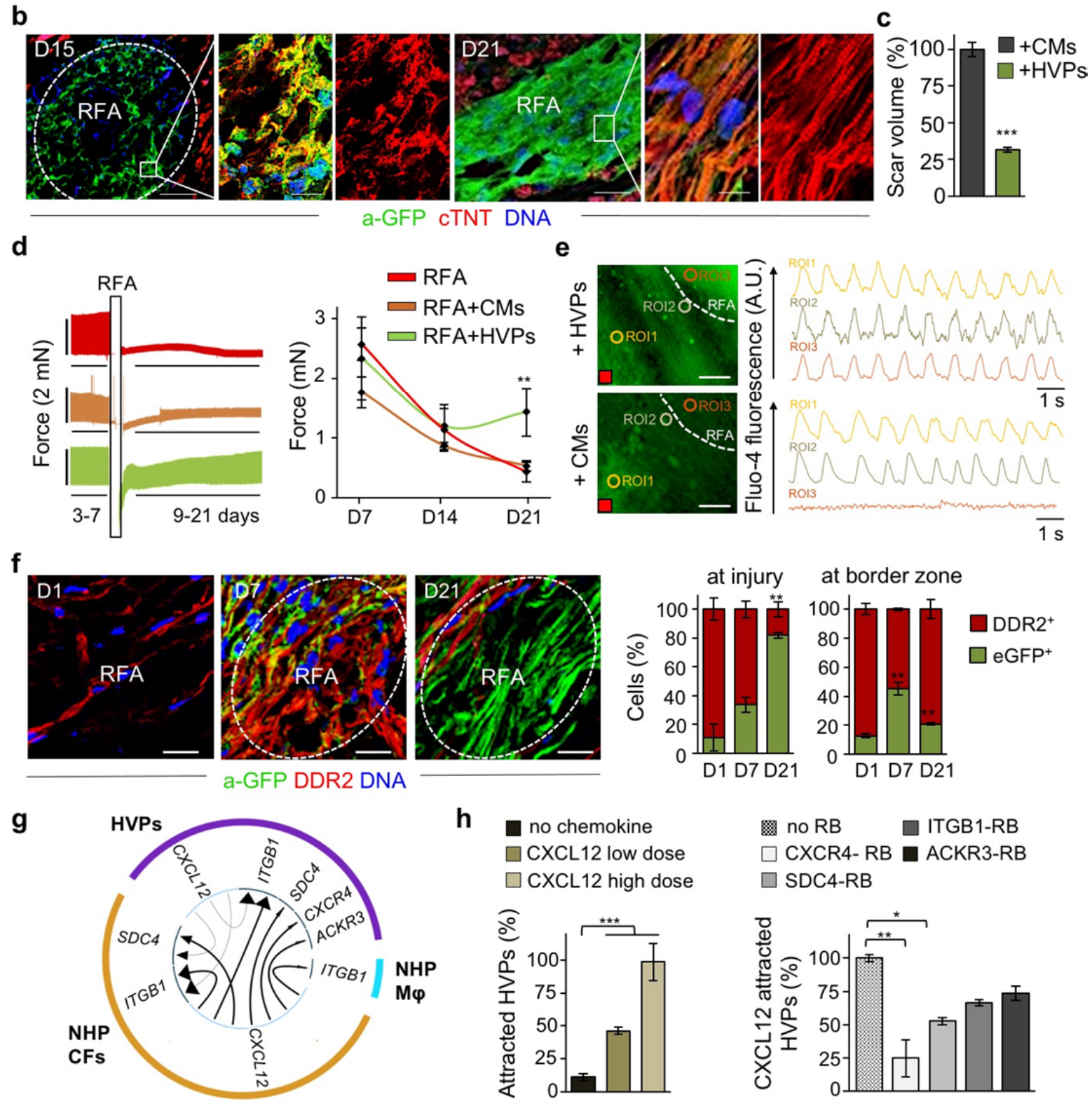

811

812 Figure 2. HVPs are chemoattracted to sites of cardiac injury via CXCL12/CXCR4

813 signalling and remuscularize the scar. a, Left, schematic of experimental design for selective 814 seeding of $N K X 2-5^{\mathrm{eGFP} / w t}$ hESC-derived HVPs or CMs onto bioprinted pluronic frame on NHP heart 
815 slices and standardized radiofrequency-ablation (RFA) injury on the opposite tissue site. Right, 816 sequential live-imaging of eGFP signal at indicated days. Scale bars $200 \mu \mathrm{m}$. b, Representative 817 immunostaining of eGFP and cardiac troponin T (cTNT) in NHP constructs on D15 and D21 after 818 RFA. Magnifications of the framed areas are shown in adjacent panels. Scale bar $200 \mu \mathrm{m}$ for D15, $819100 \mu \mathrm{m}$ for D21, $10 \mu \mathrm{m}$ for magnifications. c, Statistical analysis of relative reduction of scar volume 820 with HVPs compared to CMs on D21. $\mathrm{n}=2$ patches per group, $\geq 28 \mathrm{z}$-stack images/patch. d, Left, 821 Representative recordings of contractile force before and after RFA, separated by a blanking period of 8222 days for re-adjustment of preload (left) and corresponding statistical analysis (right). $n=3$ 823 samples/condition. e, Representative images of Fluo-4 loaded NHP-HVP and -CM constructs (left) 824 and corresponding $\mathrm{Ca}^{2+}$ transients at indicated regions of interest (ROI) (right). Scale bar $100 \mu \mathrm{m}$. Red 825 box indicate stimulation point $(1 \mathrm{~Hz})$. f, Left, representative immunostaining of eGFP and DDR2 in 826 NHP constructs at indicated days after RFA. Scale bars $200 \mu \mathrm{m}$. Right, percentage of eGFP ${ }^{+}$and 827 DDR2 $^{+}$cells at RFA injury or border zone; $n=3$ samples/time point. g, Circos plot for ligand-receptor 828 pairing showing top ten interactions identified in scRNAseq of NHP-HVP constructs at 24 and 48 829 hours after RFA injury and HVP application. Fraction of expressing cells and link direction 830 (chemokine to receptor) are indicated. $\mathrm{M} \varphi$, macrophages. h, Percentage of chemoattracted HVPs in 831 trans-well migration assays in absence and presence of low $(20 \mathrm{ng} / \mathrm{ml})$ or high $(80 \mathrm{ng} / \mathrm{ml}) \mathrm{dose}$ of 832 CXCL12 (left) or after addition of the indicated receptor blockers (right). $\mathrm{n}=3 \mathrm{samples} / \mathrm{condition}$. All data are indicated as mean $\pm \mathrm{SEM} ; * * \mathrm{p}<0.05, * * \mathrm{p}<0.005, * * * \mathrm{p}<0.001$ ( $t$-test). 
a

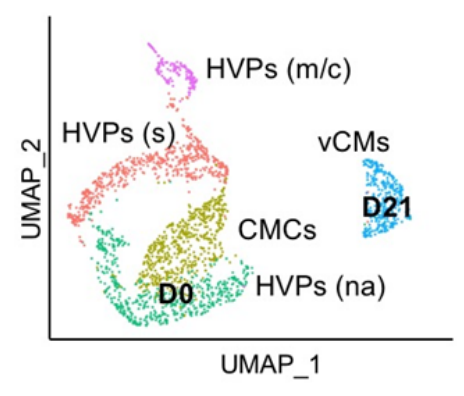

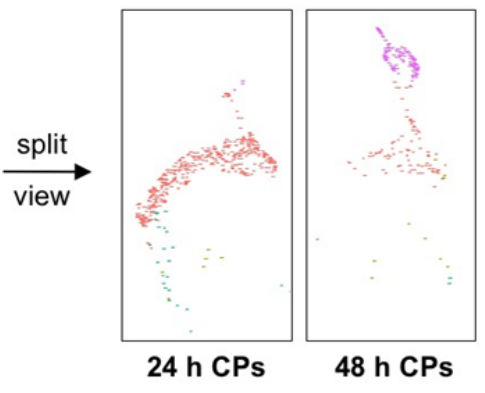

b

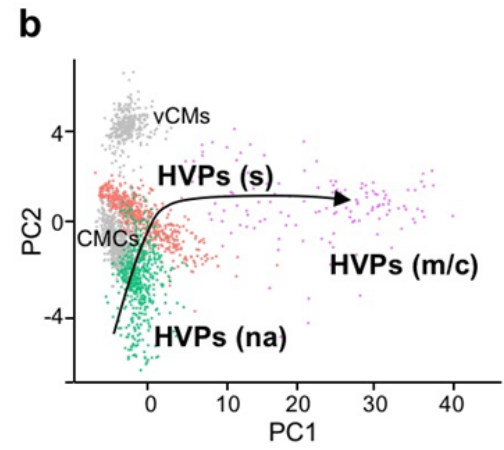

C
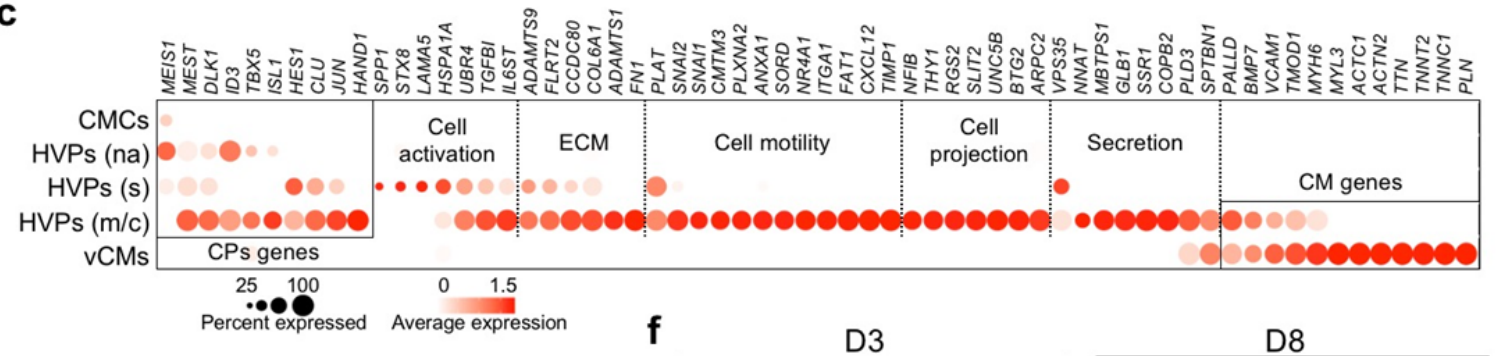

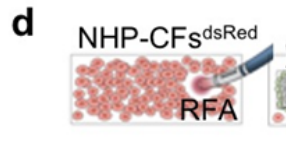

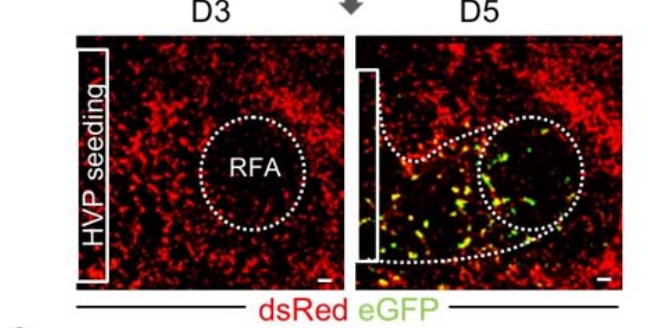

e
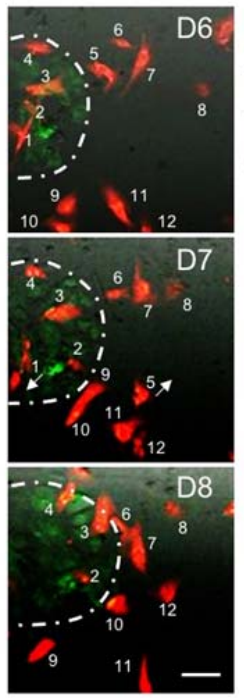

HVPs-NKX2.5 $5^{\mathrm{eGF} / w t}$

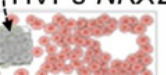
$\downarrow$
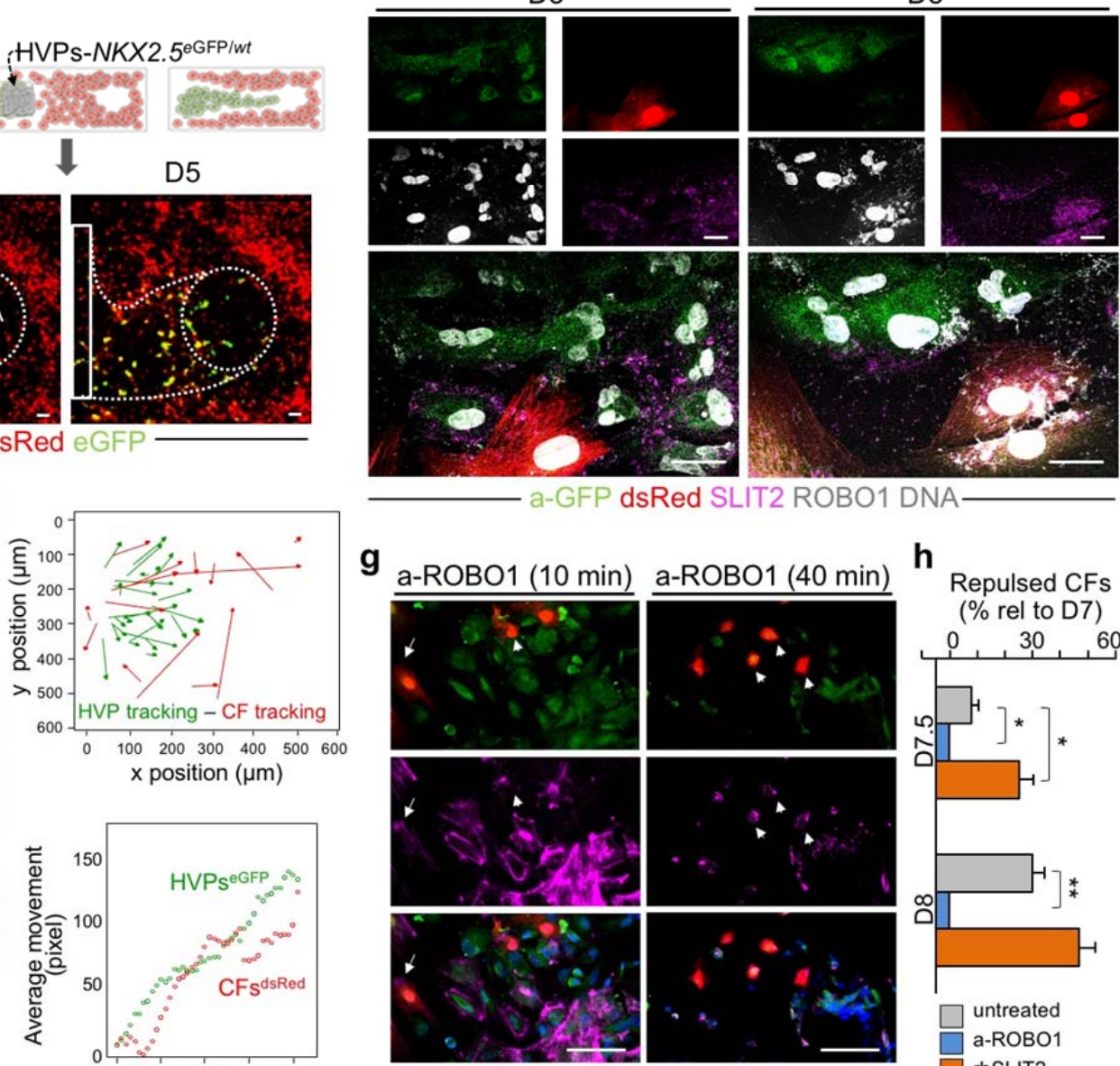

IT2 ROBO1 DNA

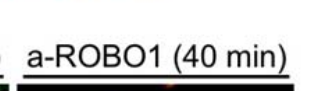

$\mathrm{h}$
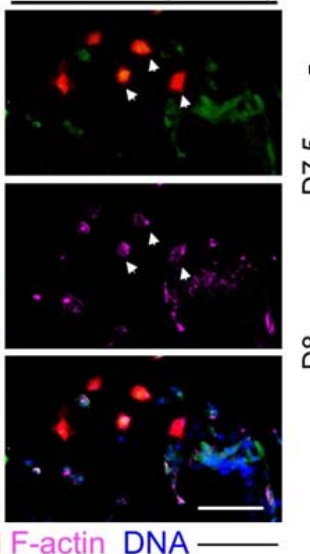

Repulsed CFs (\% rel to D7)
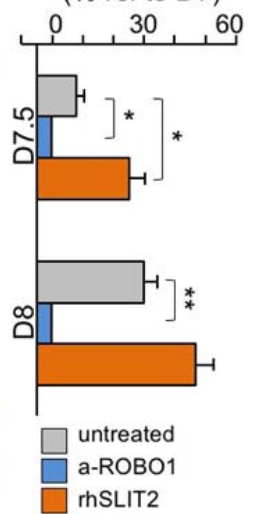

836 Figure 3. SLIT2/ROBO1 signalling mediates activated CF repulsion and prevents

837 myocardial scarring. a, Twenty-four and 48 hours human scRNAseq datasets are integrated with D0 838 and D21 CM dataset and projected onto UMAP plots, coloured by cluster assignment and annotated 
839 post hoc. Both the aligned (left) and split (right) views are shown. CMCs, cardiac mesenchymal cells; 840 HVPs (na), non-activated; HVPs (s), sensing; HVPs (m/c), migrating and counteracting; vCMs, 841 ventricle cardiomyocytes. b, PCA plot of different cell clusters, with the principal curve indicating the 842 pathway of injury response. c, Dot plot showing gene signature shifts among different dynamic 843 cellular states. The shadings denote average expression and the size of dots the fractional expression. 844 d, Top, schematic of 2D model for RFA injury of NHP-CFs expressing dsRed followed by NKX2$8455^{\mathrm{eGFP} / w t}$ HVP seeding and monitoring of co-culture. Bottom, sequential live imaging of dsRed ${ }^{+}$and $846 \mathrm{eGFP}^{+}$cells during migration. Scale bars $200 \mu \mathrm{m}$. e, Left, representative time-lapse images of dsRed ${ }^{+}$ 847 and $\mathrm{eGFP}^{+}$cells at the RFA injury site during CF repulsion on indicated days. Dotted line delineates 848 HVP migration front. Scale bar $100 \mu \mathrm{m}$. Right, cell tracking over time (top) and average movement 849 (bottom) analysis of HVPs and CFs. f, Representative immunostaining for eGFP, SLIT2, and ROBO1 850 on D3 and D8. Scale bars $25 \mu \mathrm{m}$. g, F-actin and eGFP immunofluorescence an D8 after ROBO1 851 antibody exposure for 10 and 40 minutes. Change of CF shape (arrow head) and F-actin localized on 852 protrusion side of CFs (arrow). Scale bars $75 \mu \mathrm{m}$. h, Percentage of repulsed CFs at the injured site 853 analyzed on D7.5 and D8 in standard condition (untreated) or after ROBO1 antibody and rhSLIT2 854 treatment on D7. Data are normalized to D7 and presented as mean \pm SEM, $n=3 .{ }^{*} p<0.05,{ }^{*} p<0.005$ $855 v s$ untreated ( $t$-test). 
a

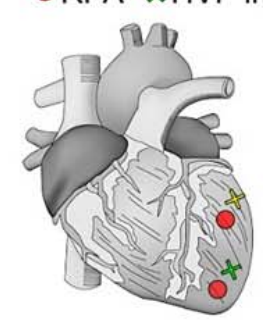

d

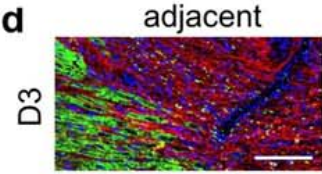

น̊
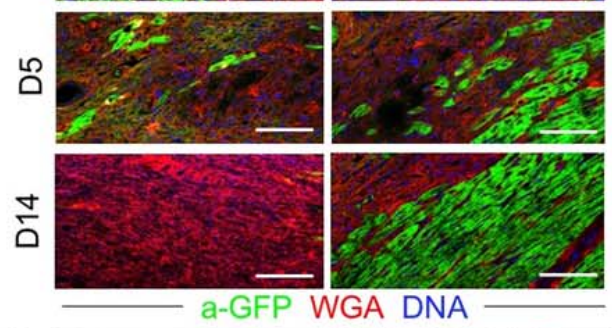

g
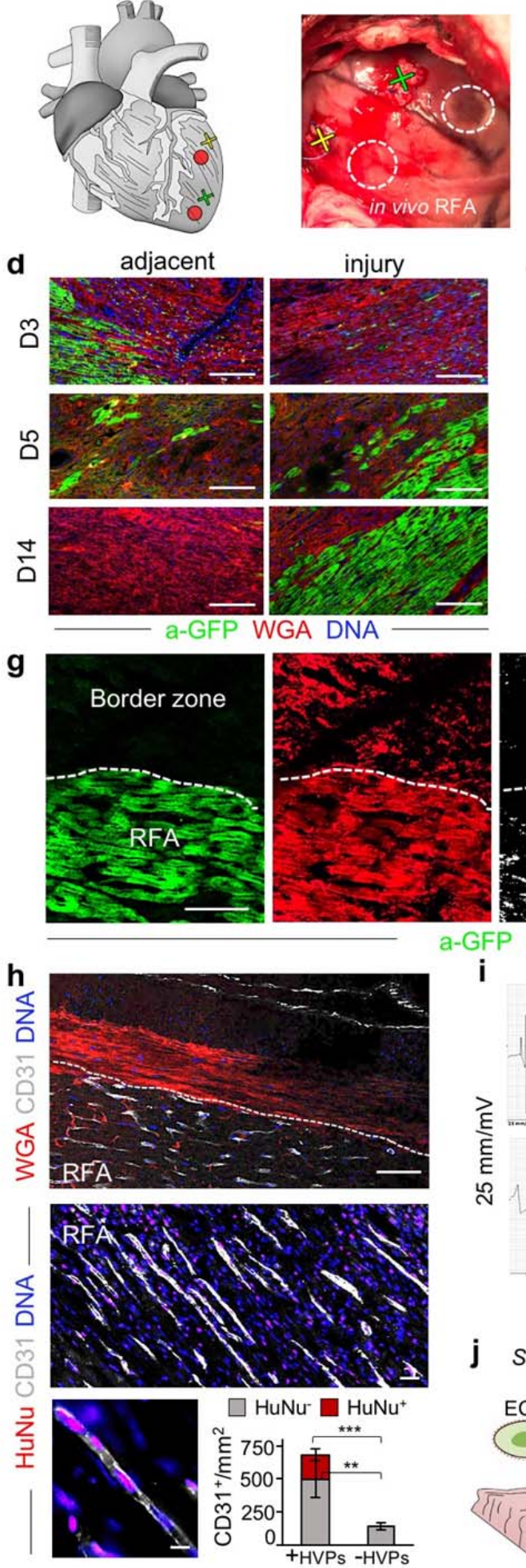
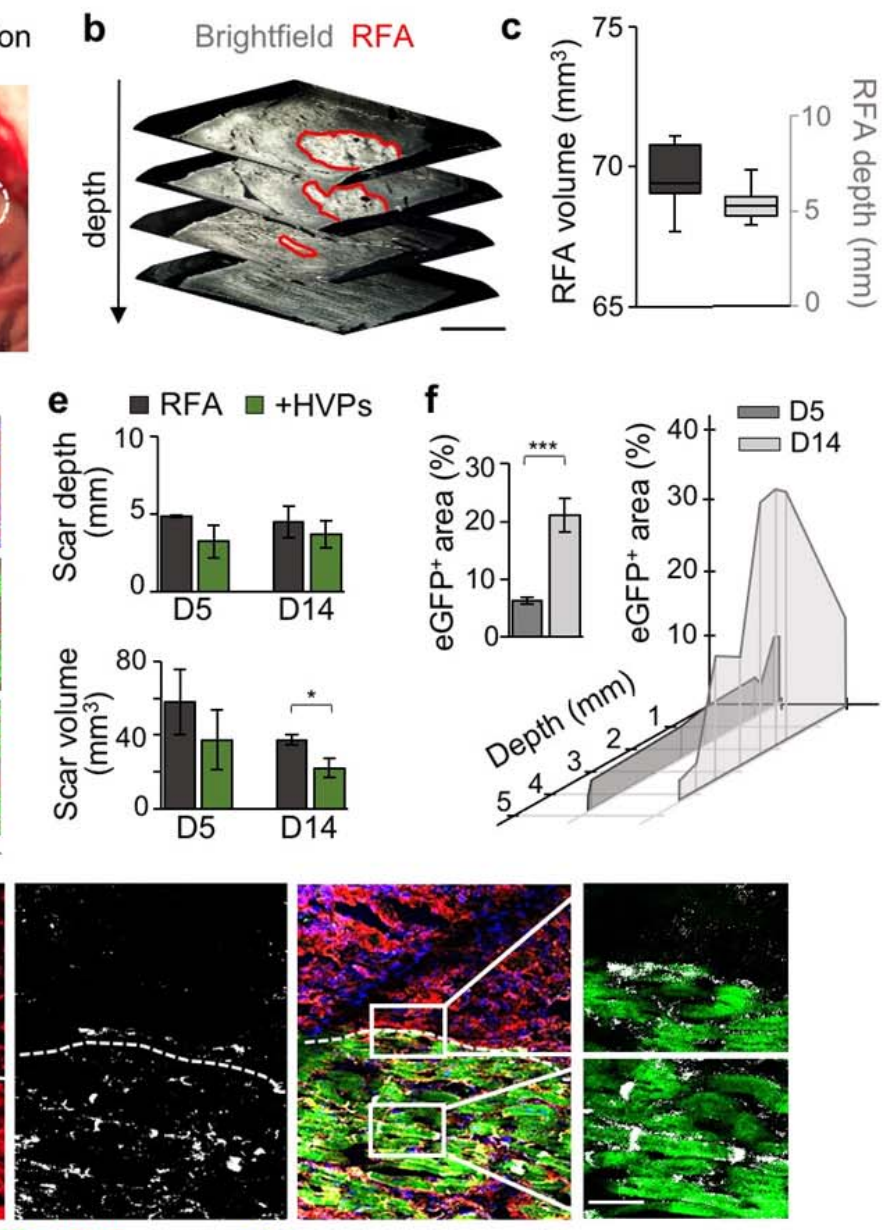

-GFP cTNT CX43 DNA
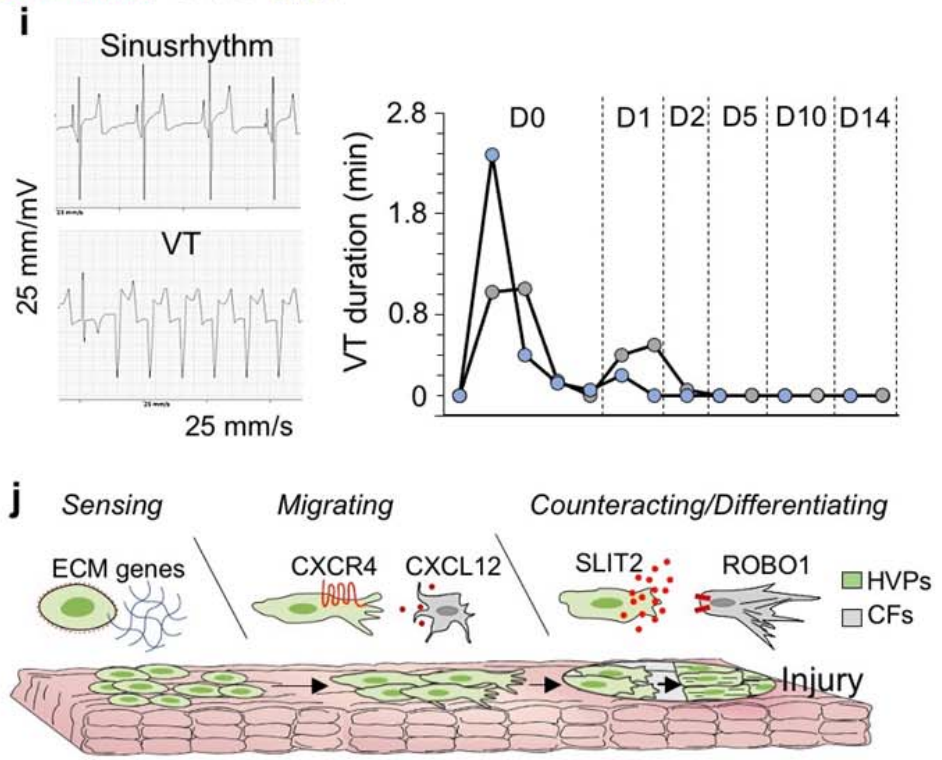

858 Figure 4. HVPs directly migrate towards the damaged myocardium and remuscularize the 859 injured tissue without arrhythmias in a transgenic LEA29Y porcine model in vivo. a, Schematic 860 of in vivo experimental design depicting 2 left ventricular RFA injuries and adjacent injection of 
HVPs or cell-free media. b, c, Representative 3D reconstruction of non-transmural RFA injury (b, scale bar $2 \mathrm{~mm}$ ) and statistical analysis of scar volume and depth of RFA injuries in freshly explanted 863 wild-type pig hearts indicating standardized injury size (c). The median and quartiles are shown; $n=3$. d, Representative fluorescence images of injury and adjacent sites after WGA and anti-GFP (a-GFP) co-staining on D3, D5 and D14. Scale bars $100 \mu \mathrm{m}$. e, Quantification of in vivo scar depth and volume on D5 and D14 with or without HVP injections. f, Percentage of GFP $^{+}$area within the RFA injury (left) and according to depth of the cutting plane. g, Representative immunostaining of eGFP, cTNT, and CX43 in RFA and border zone on D14. Magnifications on the right correspond to the boxed area in the merge image. Scale bar $50 \mu \mathrm{m}$ and $10 \mu \mathrm{m}$ (magnifications). $\mathbf{h}$, Representative fluorescence images of HVP-treated RFA injury site after immunostaining for CD31 in combination with WGA (top) or with anti-human nuclei (HuNu, middle and bottom). Scale bar $50 \mu \mathrm{m}$ top, $25 \mu \mathrm{m}$ middle, 10 $\mu \mathrm{m}$ bottom. Bar graph shows the average number of $\mathrm{CD} 1^{+}$cells $/ \mathrm{mm}^{2}$ cells derived from host $\left(\mathrm{HuNu}^{-}\right)$ or human progenitors $\left(\mathrm{HuNu}^{+}\right)$in HVP-treated and untreated RFAs. i, Eventrecorder readout with representative ECG traces of sinus rhythm (top) and degeneration to ventricular tachycardia (VT, bottom). Right, occurrence and duration of VTs at the indicated days after transplantation. n=2 eventrecorder traces. j. Scheme depicting the identified dynamical cellular states of human HVPs during tissue heart repair and the involved signalling pathways. Data in $\mathbf{e}, \mathbf{f}$, and $\mathbf{h}$ are mean $\pm \mathrm{SEM}$, $\mathrm{n}=2$ per group. $* \mathrm{p}<0.05, * * \mathrm{p}<0.005, * * * \mathrm{p}<0.001$ ( $t$-test). 
a
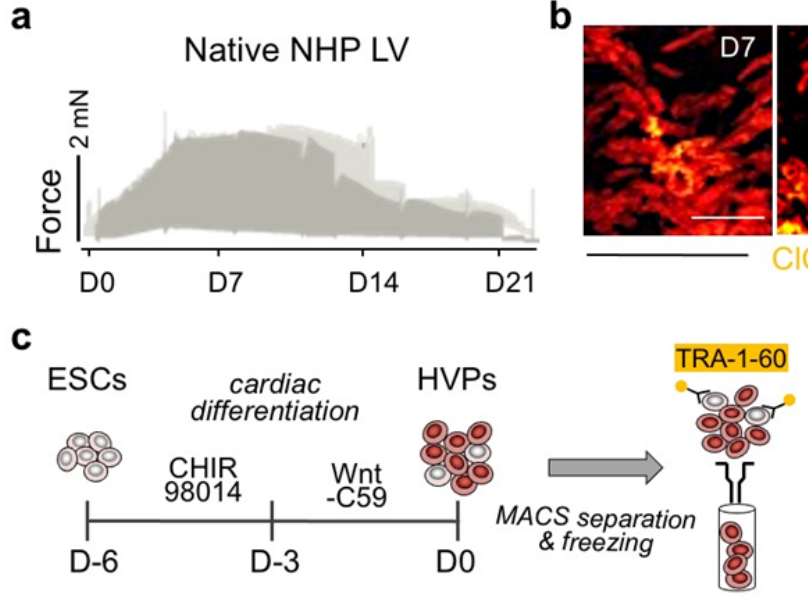

d
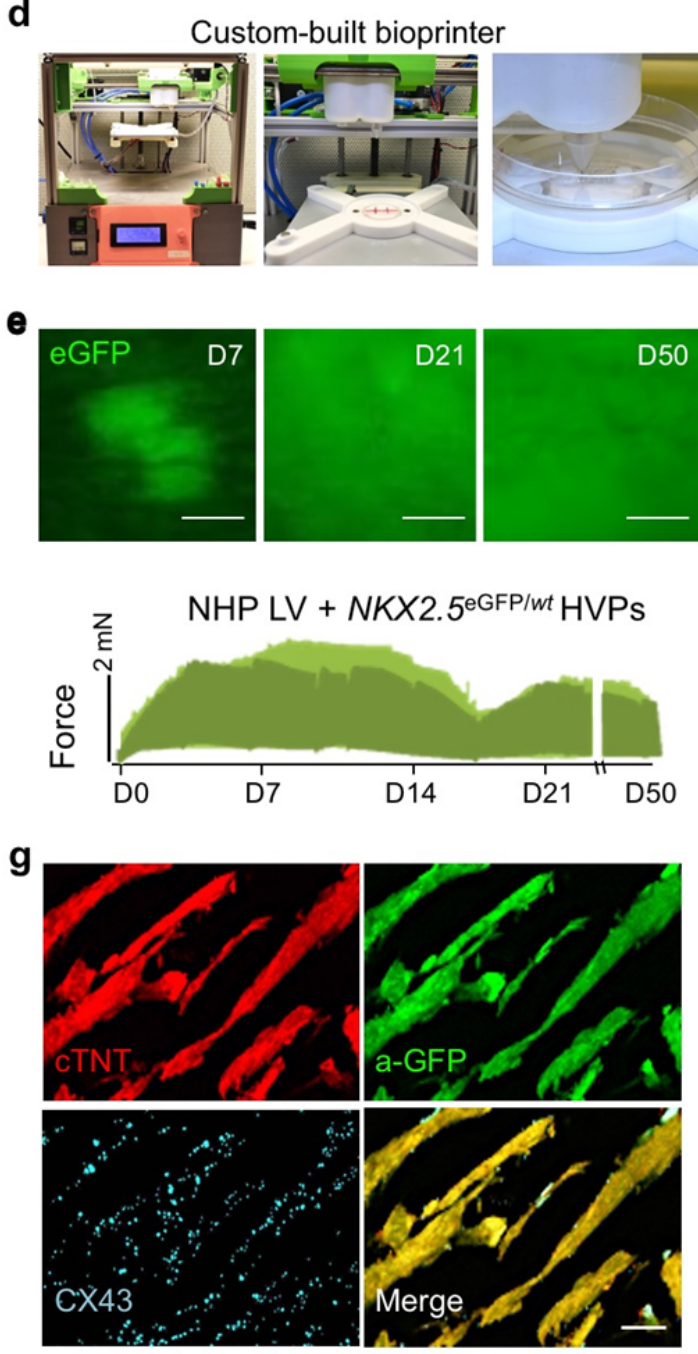
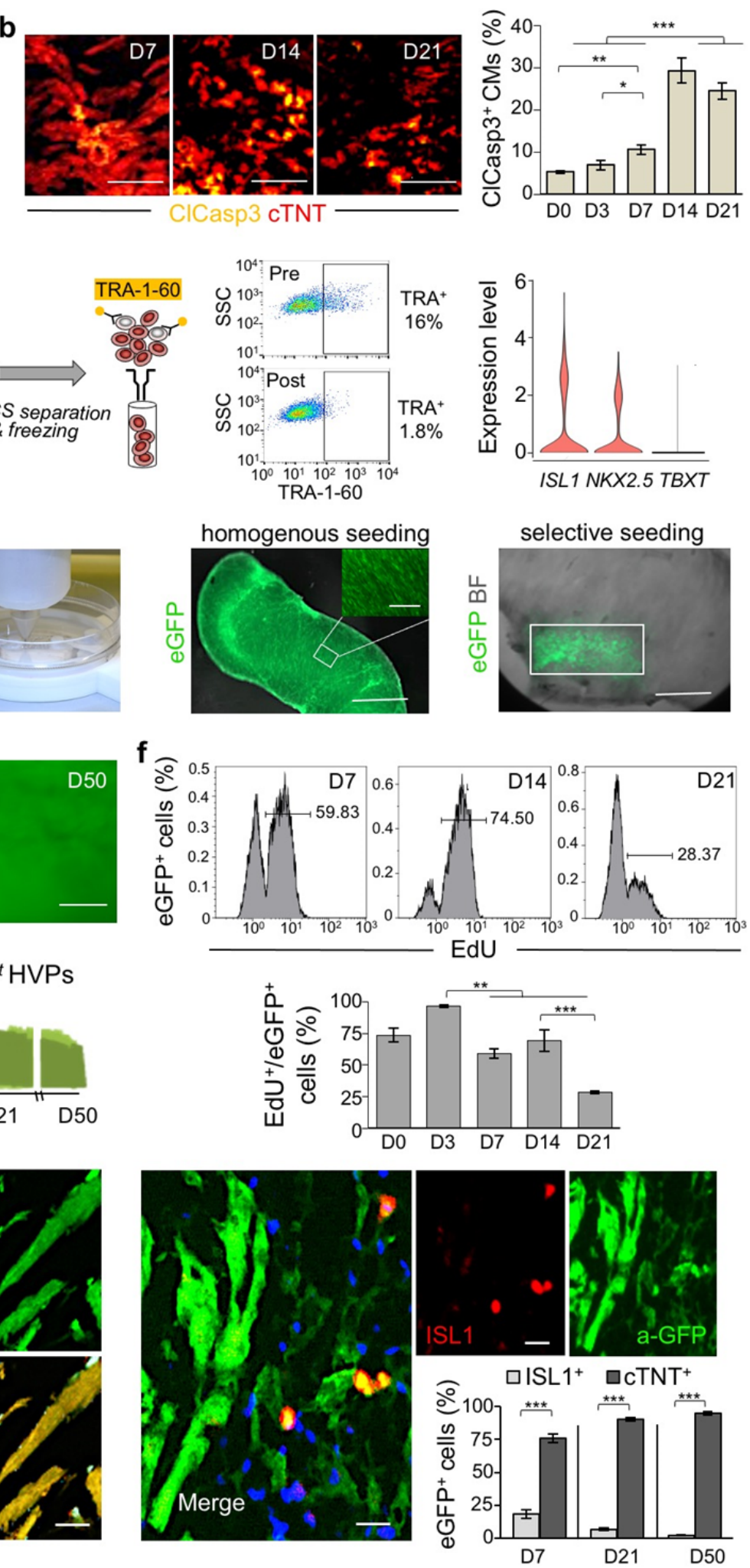

881 Extended Data Figure 1. Generation and analysis of an ex vivo 3D chimeric human-NHP

882 heart model. a, Representative, overlapped traces of contractile force of native NHP heart slices

883 cultured ex vivo for 21 days in biomimetic chambers. b, Representative immunofluorescence images 
884 for activated cleaved caspase 3 (ClCasp3) and cardiac troponin T (cTNT) in ex vivo cultured NHP 885 heart slices (left) and correspondent quantification (right) at the indicated days. Scale bars $50 \mu \mathrm{m}$. $886 \mathrm{n} \geq 3$ samples/time point. c, Schematic of ESC differentiation into HVPs by Wnt pathway modulation 887 followed by MACS depletion of Tra- $1-60^{+}$cells and cryopreservation until seeding. Single cell 888 RNAseq confirmed expression of ISL1 and NKX2.5 and loss of brachyury T (TBXT) on D0. d, Left, 889 custom-built bioprinting device with pneumatic printhead. Right, exemplary images of homogeneous 890 or selective seeding of $\mathrm{eGFP}^{+}$HVPs onto NHP heart slices by bioprinting. Scale bars $250 \mu \mathrm{m}$, inlet 75 $891 \mu \mathrm{m}$. e, Live eGFP imaging of NHP heart slices after $N K X 2-5^{\mathrm{eGFP} / \omega t} \mathrm{HVP}$ seeding at the indicated days 892 of co-culture (top) and representative contractile force traces (bottom). f, Flow cytometry analysis for 893 EdU in $\mathrm{eGFP}^{+}$cells isolated at the indicated days of co-culture. $\mathrm{n}=3$ samples/time point. g, 894 Immunostaining of eGFP in combination with cTNT and Connexin-43 (CX43) (left) or ISL1 (right) 895 on D50 of co-culture. Scale bars $25 \mu \mathrm{m}$. Bar graph shows the percentage of eGFP ${ }^{+}$cells expressing 896 ISL1 and cTNT at the indicated days of co-culture.

897 All statistical data are shown as mean \pm SEM; $* \mathrm{p}<0.05, * * \mathrm{p}<0.005, * * * \mathrm{p}<0.001$ ( $t$-test). 

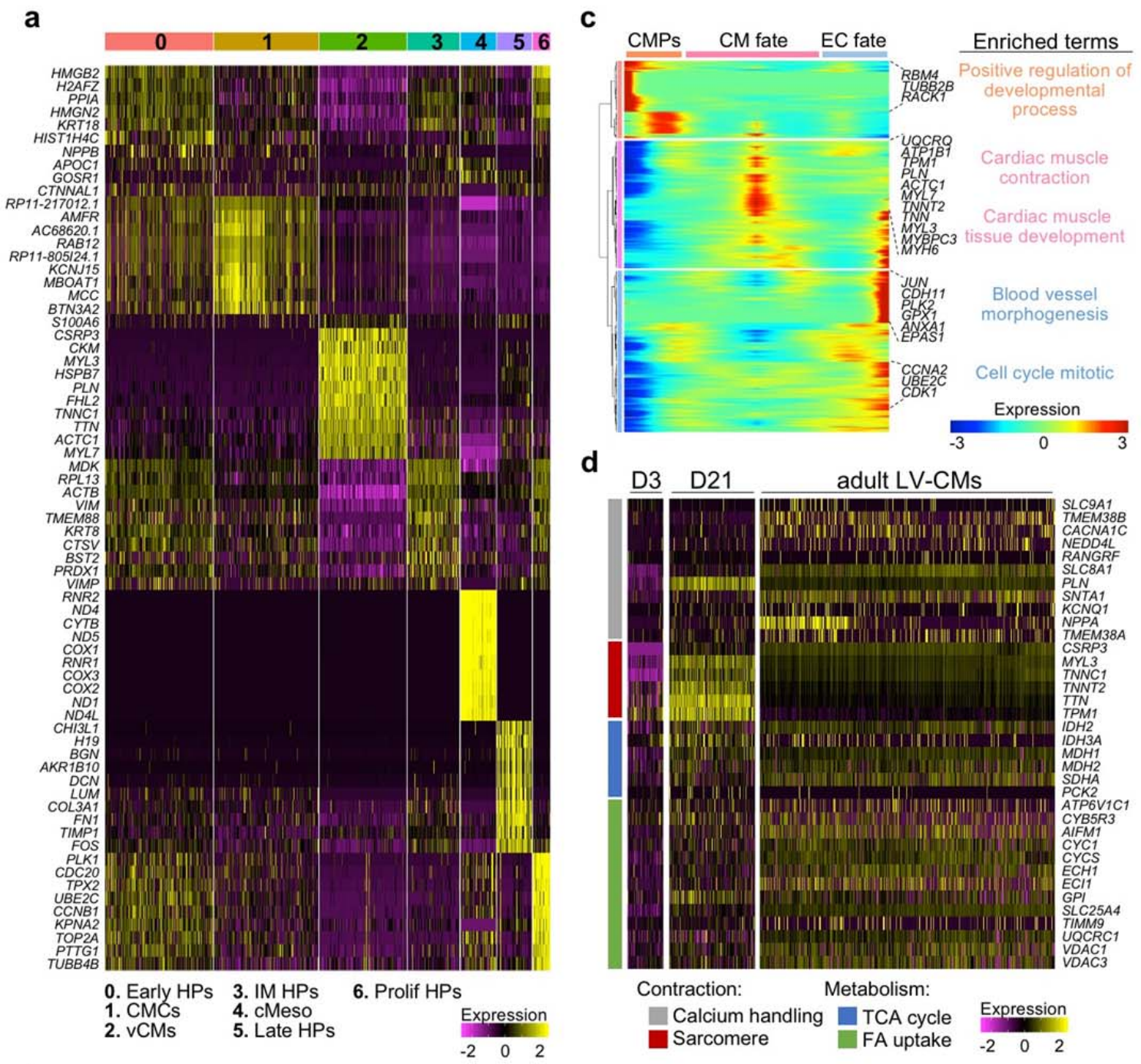

b

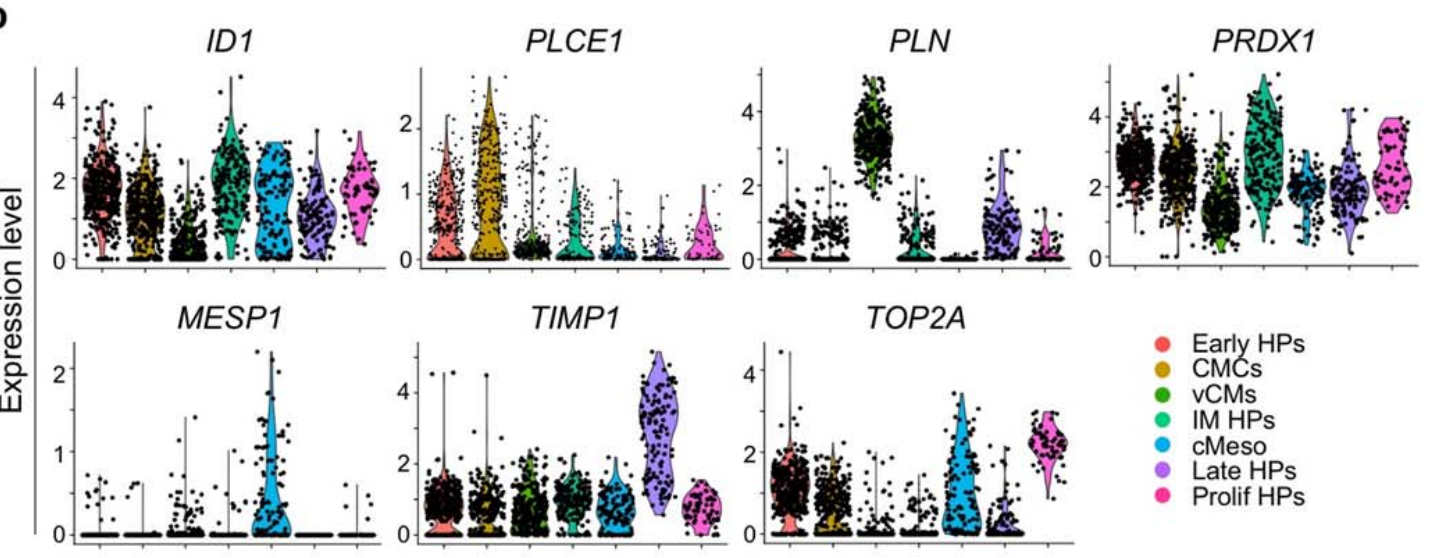

900 Extended Data Figure 2. scRNAseq analysis of human $N K X 2.5^{\mathrm{eGFP} / w t} \mathrm{HVPs}$ in a chronic 901 injury model of NHP heart slices. a, Heatmap showing expression of the top 10 genes in each 902 cluster defined as 0 - early heart progenitors (Early HPs), 1- cardiac mesenchymal cells (CMCs), 2- 
903 ventricular cardiomyocytes (vCMs), 3- intermediate heart progenitors (IM HPs), 4- cardiac 904 mesodermal cells (cMeso), 5- late heart progenitors (Late HPs), 6- proliferating heart progenitors 905 (Prolif HPs). b, Violin plots of cluster specific marker genes, $p$-value $\leq 0.05$. c, Heatmap of different 906 blocks of DEGs along the pseudotime trajectory and representative genes in each cluster. Cardiac 907 mesodermal precursors (CMPs, D-3), endothelial cell (EC) fate (D0 and D3) and CM fate (D21). 908 Selected top biological process and canonical pathway terms related to corresponding DEGs. d, 909 Heatmap showing the expression of genes related to contraction (gray and red) and metabolism (blue 910 and orange) in eGFP ${ }^{+}$cells on D3 and D21 of ex vivo co-culture compared to adult human LV-CMs 911 (Wang et al., 2020). Expression levels are presented as a colour code. 
a
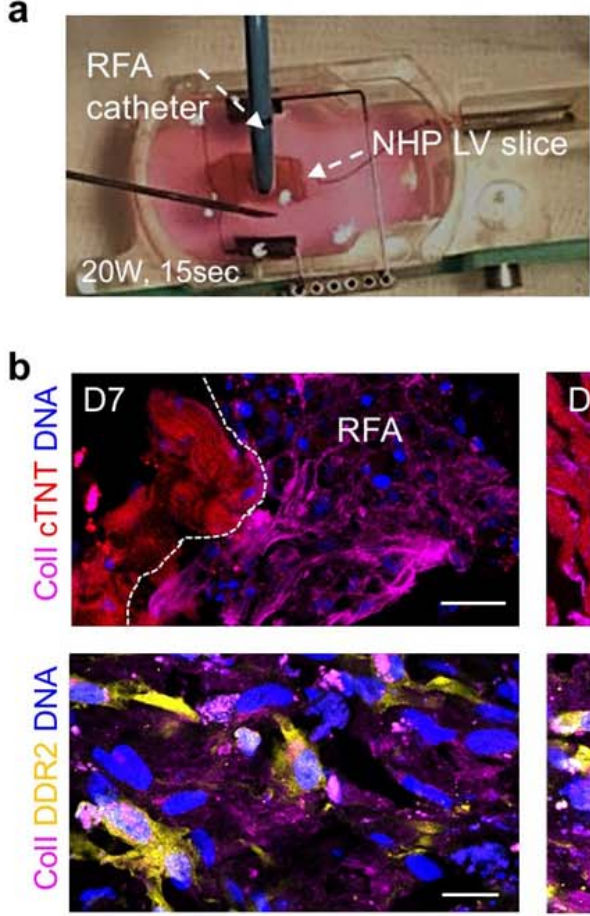

C
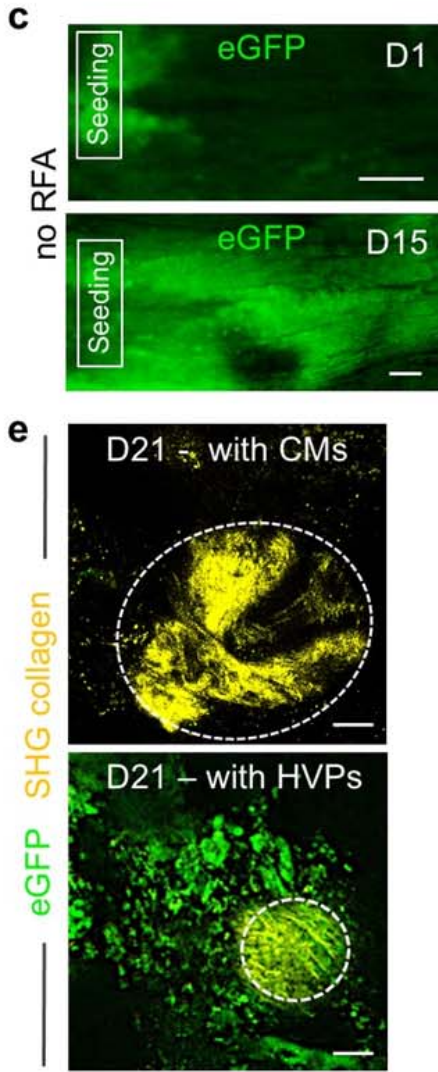

d

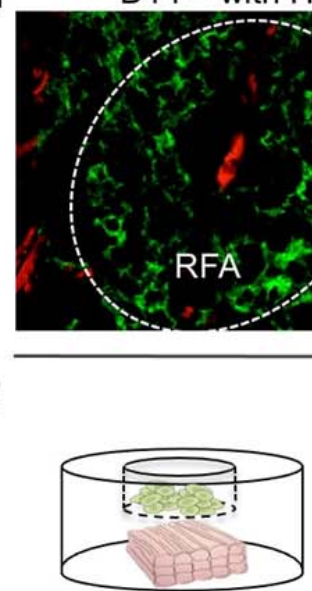

no RFA

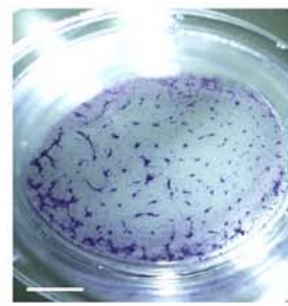

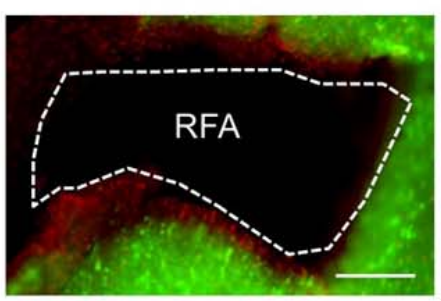

Ethidium Dimer Calcein

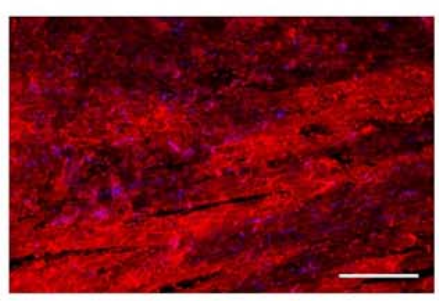

WGA DNA
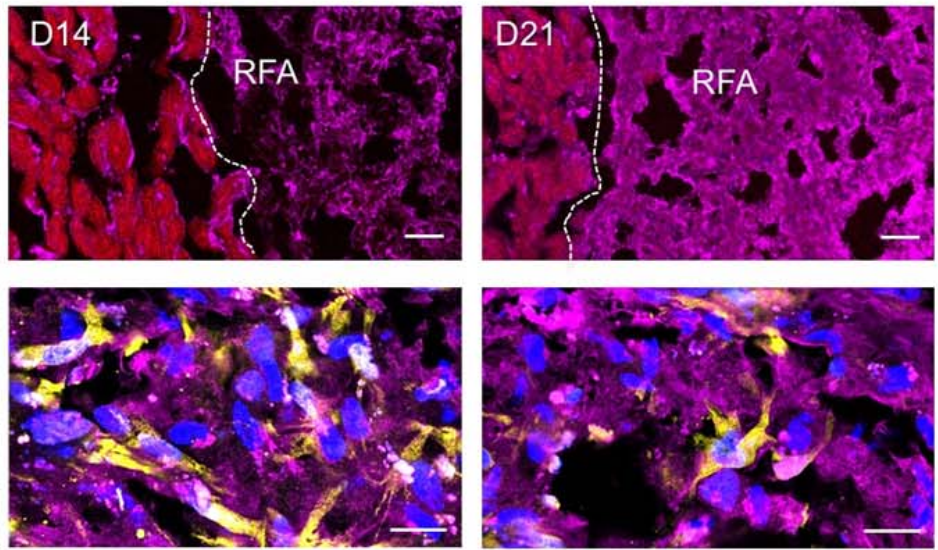

D14 - with CMs

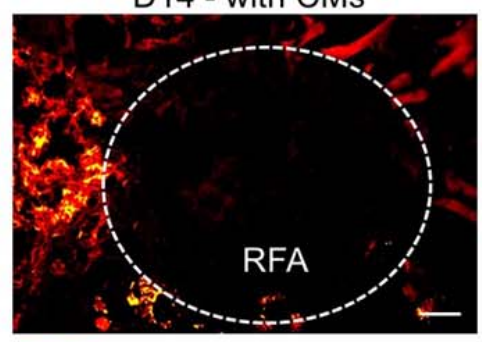

cTNT
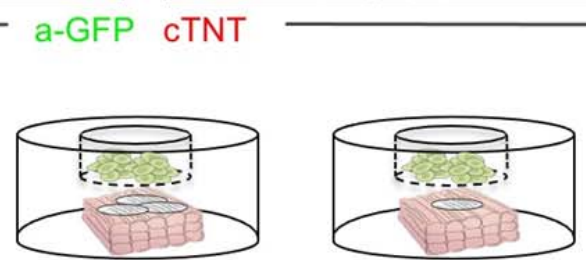

multiple RFAs

single RFA
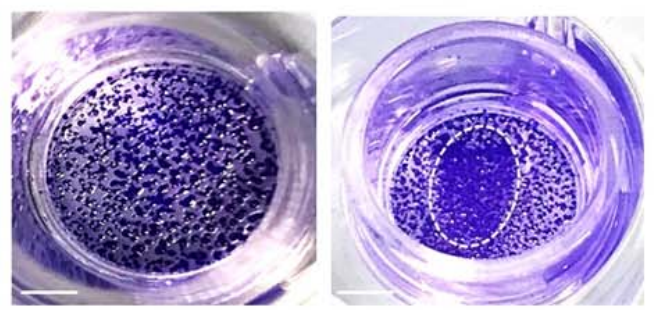

migrated cells

914 Extended Data Figure 3. Generation and analysis of an acute ex vivo NHP heart injury

915 model. a, Standardized non-transmural myocardial injury in NHP heart slices by defined RFA. Live

916 and dead cells are stained by calcein and ethidium dimer, respectively (middle). ECM fibers are 
917 labeled by WGA (right). Stainings were performed immediately after RFA. Scale bar $200 \mu \mathrm{m}$. b, 918 Representative fluorescence images of RFA-injured slices after immunostaining for Collagen type I 919 (ColI) combined with cTNT (top) or DDR2 (bottom) on indicated days. Lower panels show images of 920 the RFA area. Scale bars $30 \mu \mathrm{m}$ (top) and $25 \mu \mathrm{m}$ (bottom). c, Sequential live imaging of NKX2$9215^{\mathrm{eGFP} / w t}$ HVPs migrating from the seeding frame into the tissue showing homogenous repopulation of 922 the slice by D15 in the absence of RFA injury. Scale bars $200 \mu \mathrm{m}$. d, Representative immunostaining 923 of eGFP and cTNT in RFA-injured area on D14 after selective seeding of $N K X 2-5^{\mathrm{eGFP} / w t}$ HVPs (left) 924 or CMs (right). Scale bars $50 \mu \mathrm{m}$. e, Two-photon live microscopy of RFA-injured slices for eGFP and 925 second-harmonic-imaging (SHG) visualization of collagen and scar size on D21. Circles demarcate 926 areas with collagen deposition. Scale bars $100 \mu \mathrm{m}$. f, Trans-well migration assays with D0 NKX2$9275^{\mathrm{eGFP} / w t}$ HVPs in the upper and NHP heart slice in the lower compartment, respectively. Images show 928 trans-well migrated HVPs on polycarbonate membrane in the absence (left) or presence of multiple 929 (middle) or single (right) RFA injury. Dashed line marks the site of HVP accumulation. Scale bars 2 $930 \mathrm{~mm}$. 
a

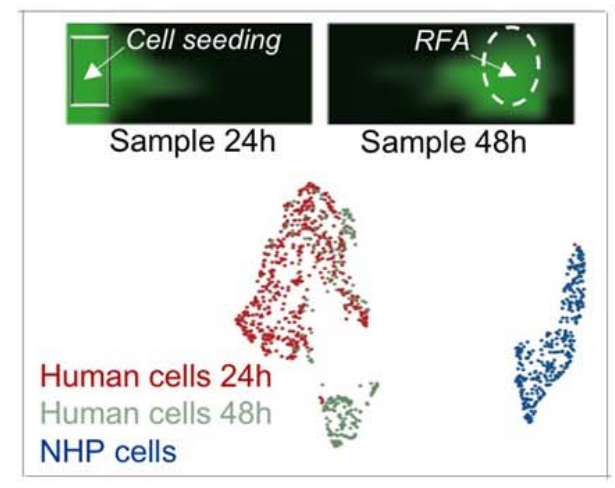

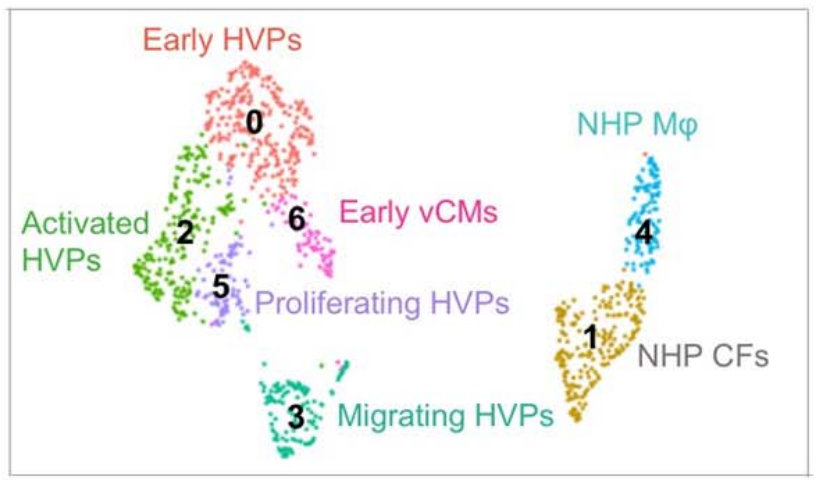

b

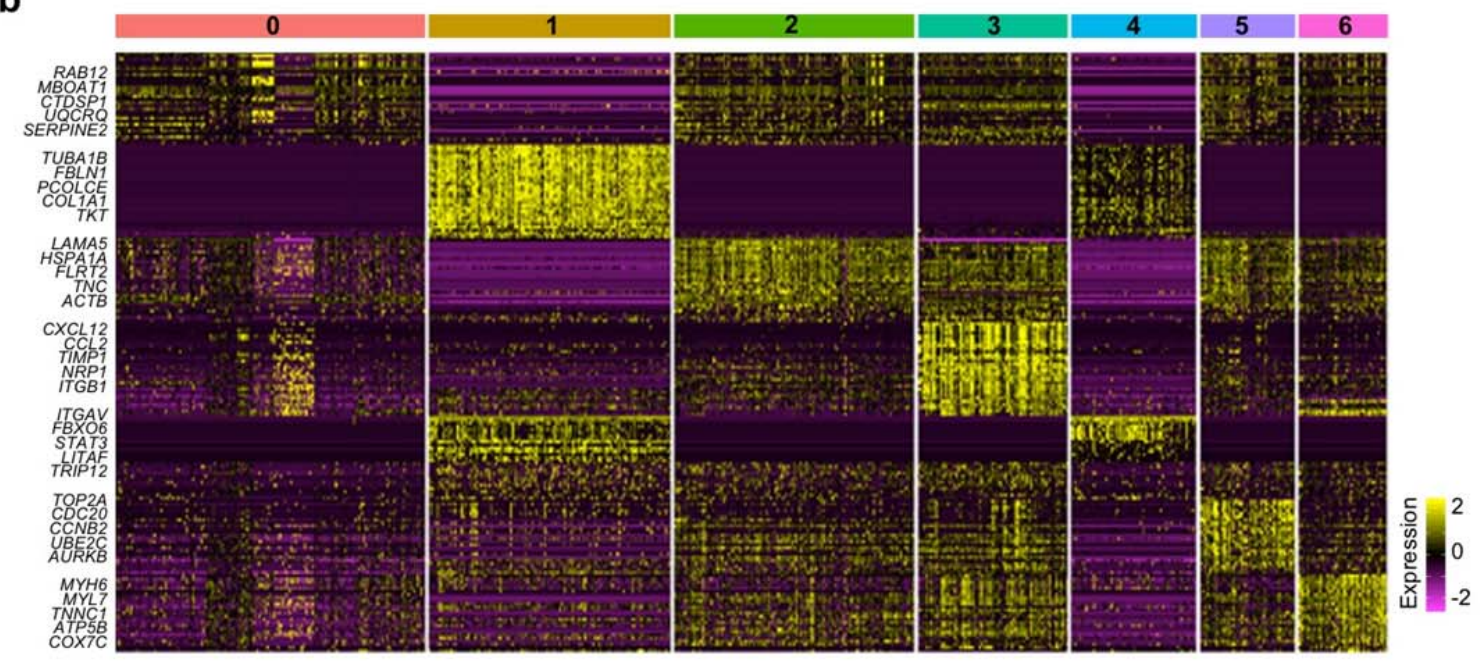

C

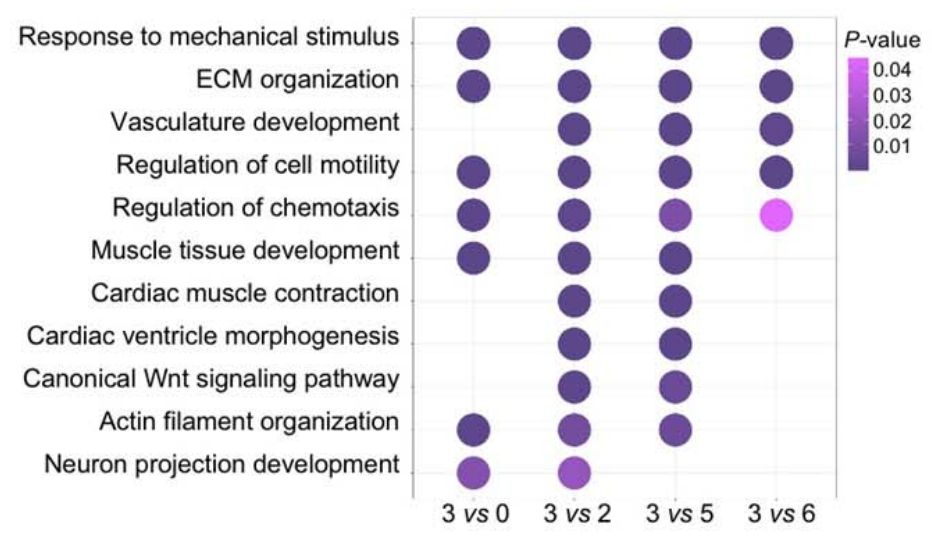

d

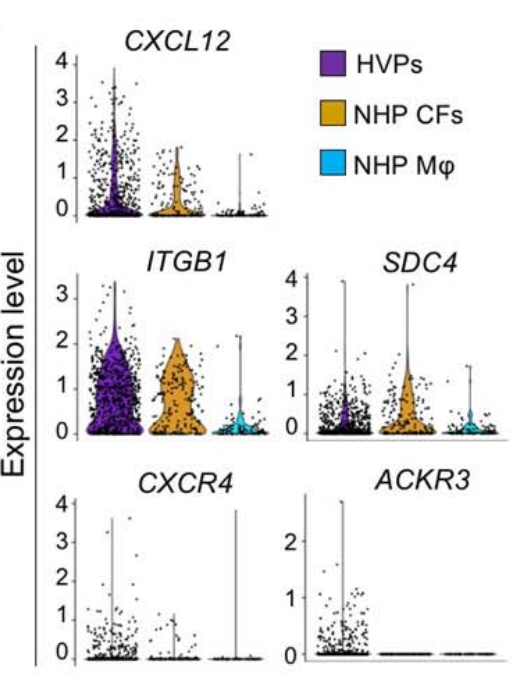

933 Extended Data Figure 4. scRNAseq analysis of human $N K X 2.5^{\mathrm{eGFP} / \mathrm{wt}} \mathrm{HVPs}$ and NHP

934 cardiac cells after acute RFA heart injury. a, Left, representative images of HVPs seeded on an 
935 injured NHP heart slice at the time points used for cell collection (24h and 48h) (top) and UMAP plot 936 of all captured cells (bottom). Right, relative UMAP clustering of captured cells. NHP, non-human 937 primate; RFA, radiofrequency ablation; HVPs, human ventricular progenitors; vCMs, ventricular 938 cardiomyocytes; $\mathrm{M} \varphi$, macrophages. b, Heatmap of top 50 genes in each cluster with representative 939 genes indicated. c, Representative GO terms upregulated in cluster 3 (migrating HVPs) compared to 940 the other human clusters $(0,2,5,6)$. d, Violin plots of CXCL12 and its binding targets in HVPs, NHP 941 CFs and NHP M $\varphi$. 
a

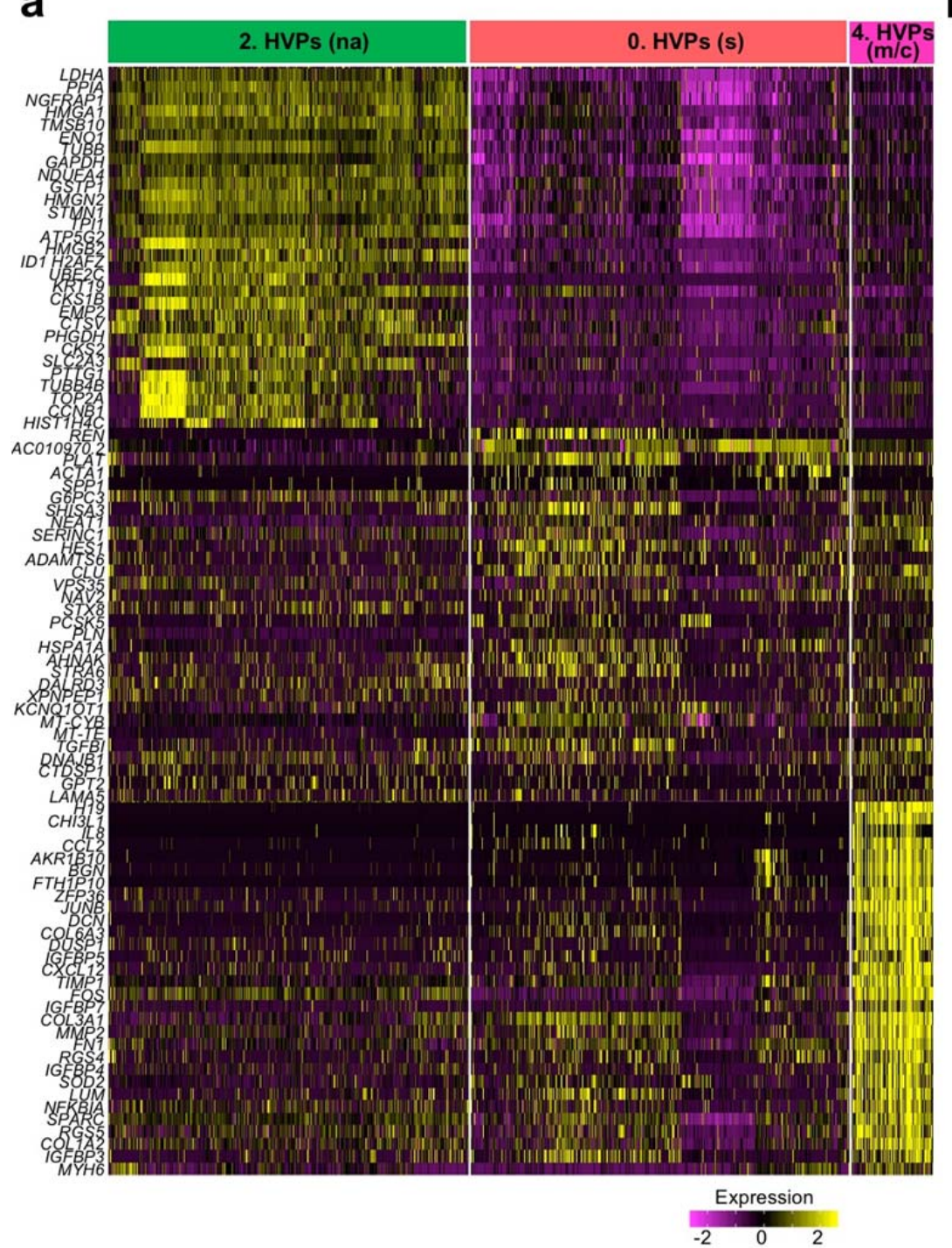

b RFA+HVPs vs RFA

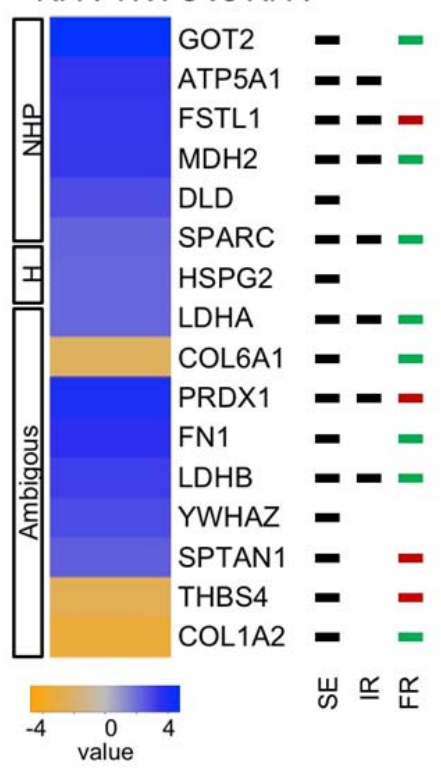

SE: Secretion Evidence

IR: Involved in Inflammatory Response FR: Fibrotic Response

- Pro = Anti

944 Extended Data Figure 5. Gene signatures of dynamical cardiac progenitor states and 945 proteomic analysis of secretome during acute injury response. a, Heatmap of top 30 genes 946 depicting the expression of DEGs in non-activated HVPs (cluster 2), sensing HVPs (cluster 0), and 947 migrating/counteracting HVPs (cluster 4). b, Proteomic analysis of supernatant of injured NHP heart 948 slices with and without application of HVPs at 48h after RFA. NHP, H, and ambiguous, was assigned 949 to proteins for which the majority of identified peptides belonged to protein sequences of macaca 950 fascicularis, homo sapiens, or both species, respectively. $\mathrm{n}=3$ biological replicates per group, $p$-value $951 \leq 0.05$. 
a
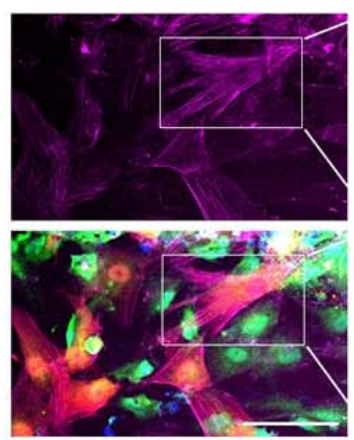

a-GFP dsRed F-actin DNA b

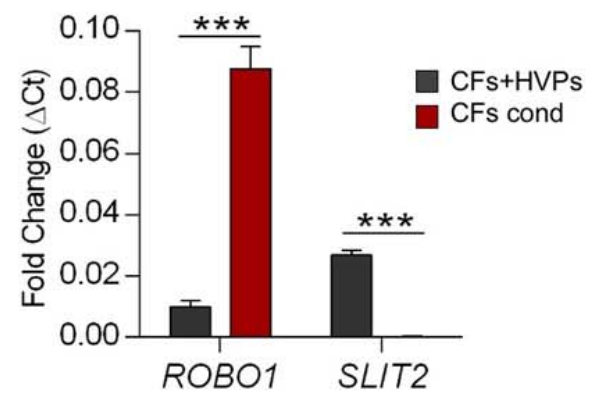

C

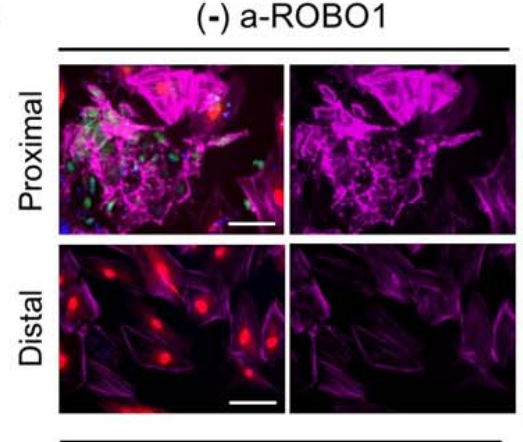

(+) a-ROBO1 (10min)
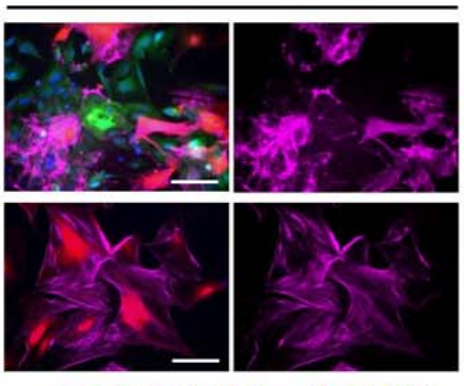

a-GFP dsRed F-actin DNA
(+) a-ROBO1 (40min)
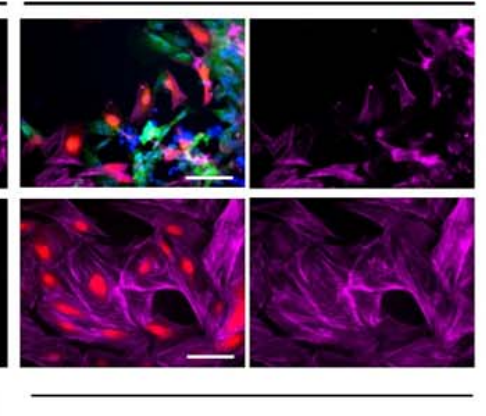

d

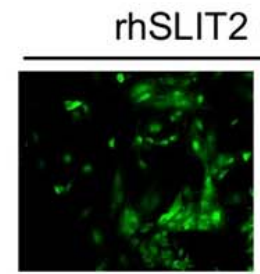

(10 min)
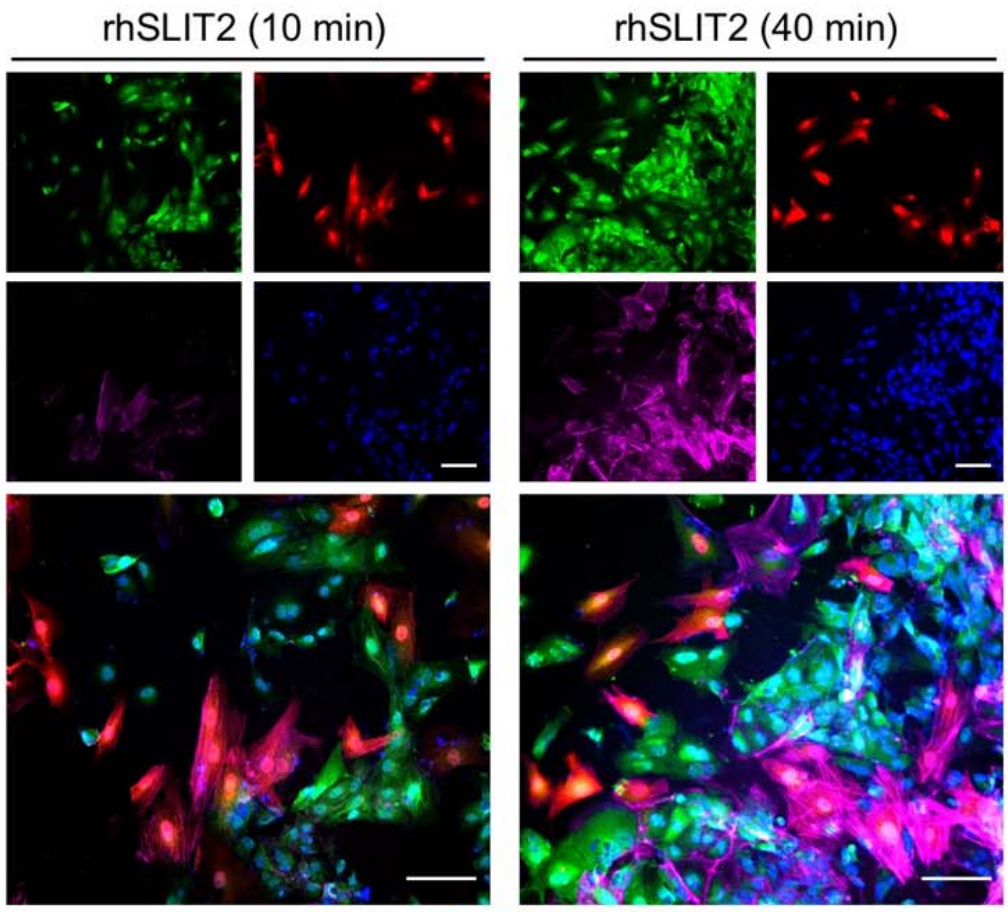

a-GFP dsRed F-actin DNA

954 Extended Data Figure 6. Analysis of CF repulsion signaling during acute injury response in 955 2D monolayer. a, Representative images of eGFP ${ }^{+}$HVPs and dsRed ${ }^{+}$CFs after F-actin staining

956 during the repulsion phase in the injury area on D8. b, Quantitative RT-PCR analysis of ROBO1 and 
957 SLIT2 expression in injured CFs cultured with HVPs (CFs+HVPs) or alone in conditioned medium 958 from HVP-CF co-culture (CFs cond) on D8. Data are mean $\pm \mathrm{SEM}, \mathrm{n}=2 .{ }^{* * *} p<0.001$ ( $t$-test). c, 959 Representative F-actin immunostaining on D8 in standard condition and after ROBO1 antibody 960 exposure for 10 and 40 minutes showing CFs in contact with HVPs (proximal) and CFs in the remote 961 area from the injury site (distal). d, Immunodetection of eGFP in conjunction with Phallodin (F-actin) 962 stain in HVP-CF co-culture on D8 after recombinant human SLIT2 (rhSLIT2) exposure for 10 and 40 963 minutes. Nuclei were counterstained with Hoechst and CFs are labelled with dsRed (a, c, d). Scale 964 bar, $75 \mu \mathrm{m}(\mathbf{a}, \mathbf{c}, \mathbf{d})$. 
a

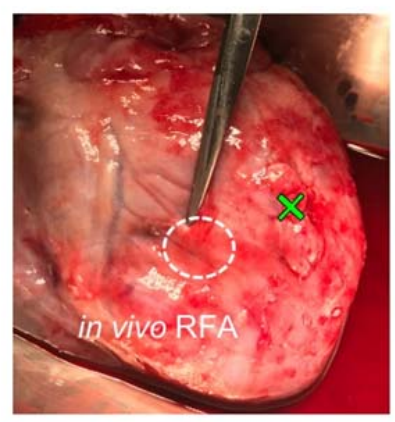

× HVP injection site b
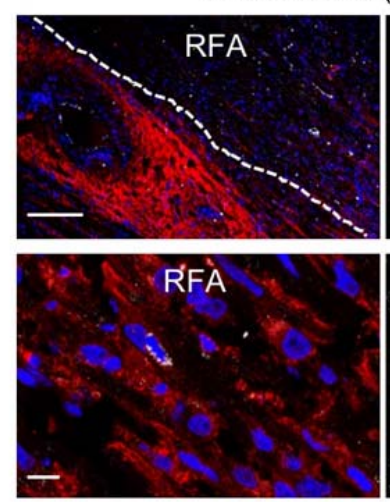

WGA CD31 DNA

C
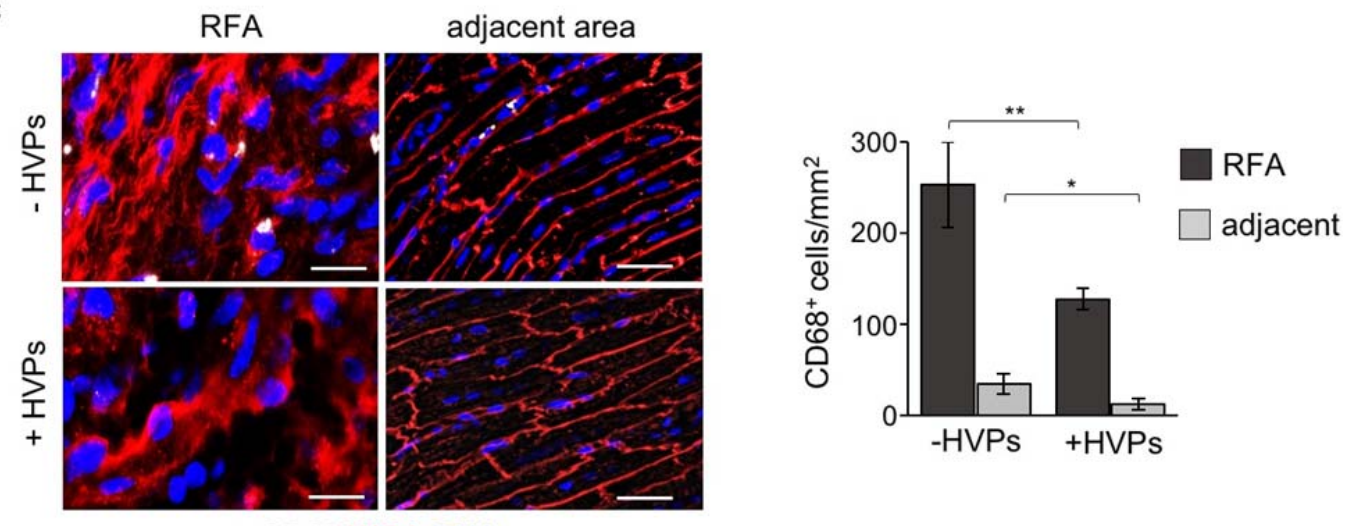

Extended Data Figure 7. Macro- and microscopic analyses of LEA29Y pig hearts after RFA injury and HVP injection in vivo. a, Image of a freshly explanted LEA29Y pig heart 14 days after in vivo RFA and adjacent HVP injection showing no macroscopic signs of teratoma formation. $\mathbf{b}$, Representative fluorescence images of control RFA and adjacent area (top) or magnified zoom of control RFA (bottom) after CD31 immunodetection and WGA labelling. Scale bars $100 \mu \mathrm{m}$ (top) and $10 \mu \mathrm{m}$ (bottom). c, Representative immunofluorescence stainings of CD68 (left) and correspondent statistical analysis (right) in RFA and adjacent areas in the presence and absence of HVPs. Scale bars $25 \mu \mathrm{m}$. Data are shown as mean $\pm \mathrm{SEM}, \mathrm{n}=7$ slices from 2 pigs per group. ${ }^{*} \mathrm{p}<0.05,{ }^{* *} \mathrm{p}<0.005(t-$ 975 test). 


\section{Supplementary Information}

979 Supplementary Table 1. Data source for scRNAseq and proteomic analyses

980 Supplementary Table 2. List of antibodies, fluorescent probes, recombinant proteins, and assays 981 used in the study

982 Extended Data Movie 1. Time-lapse live imaging of HVPs (green) and NHP CFs (red) at the injury 983 site (frame time: 90 minutes, duration: 3 days)

984 Extended Data Movie 2. RFA and cell transplantation procedure in LEA29Y porcine hearts 